\title{
Acute Stress Increases Risky Decisions and Dampens Prefrontal Activation Among
}

\section{Adolescent Boys}

\author{
Jessica Phuong \& Adriana Galván \\ Department of Psychology \\ University of California, Los Angeles
}

Corresponding Author:

Adriana Galván, $\mathrm{PhD}$

1285 Franz Hall, Box 951563

University of California, Los Angeles,

Los Angeles, CA 90095

agalvan@ucla.edu

Acknowledgements: This research was supported by a grant to AG from the National Science Foundation (BCS 0963750) and by the Center for Translational and Prevention Science (P30 DA027827, Brody-PI) funded by the National Institute on Drug Abuse 


\begin{abstract}
Adolescence is characterized by increased risky decision-making, enhanced mesolimbic response to risk and reward, increased perceived stress, and heightened physiological response to stress relative to other age groups. In adults, evidence suggests that acute stress increases risky decision-making by stress-induced increases of dopamine in regions implicated in reward processing and decision-making. Acute stress also increases risky decision-making in adolescents, but the underlying neurobiological mechanisms remained unexplored. In this study, daily self-reports of stress were documented in adolescents and adults. Participants completed two fMRI visits during which they performed a risky decision-making task: once each when they endorsed a high and low level of stress. Results revealed that adolescent males took more advantageous risks under high stress relative to low stress whereas adult males took fewer nonadvantageous risks under high stress relative to low stress. Adolescent males also showed a stress-related decrease in prefrontal activation when making risky decisions from high stress to low stress while adult males maintained prefrontal activation when making risky decisions across stress conditions. Adolescent and adult females did not exhibit stress-related changes in risky decisions. Moreover, greater prefrontal activation under stress was associated with fewer nonadvantageous risks taken under stress. Implications for risk-taking under stress are discussed in light of these findings.
\end{abstract}




\section{Acute Stress Increases Risky Decisions and Dampens Prefrontal Activation Among Adolescent Boys}

Adolescence is a developmental period characterized by increased real-life (Dahl, 2004) and laboratory-based (Burnett, Bault, Coricelli, \& Blakemore, 2010; van Leijenhorst et al., 2010) risky decision-making. It is also marked by increased stress and heightened reactivity to acute stress, compared to children and adults (Dahl \& Gunnar, 2009). Surprisingly, how acute stress influences decision-making and associated neural functioning in adolescents remain poorly understood. Addressing this question is critical because (1) heightened acute stress may exacerbate risky decision making in adolescence and (2) neural systems that are vulnerable to the effects of stress in adults undergo significant neurodevelopment during adolescence.

In animals, activation of the hypothalamic-pituitary-adrenal (HPA) axis in response to acute stress releases glucocorticoids that bind to glucocorticoid receptors (GR) on dopaminergic cells in the ventral tegmental area (VTA), which releases dopamine onto the nucleus accumbens (NAcc) of the ventral striatum and prefrontal cortex (PFC) (Abercrombie, 1989; Rougé-Pont, Deroche, Le Moal, \& Piazza, 1998). There is an inverted U-shape pattern between D1 receptor binding in the PFC and cognitive function such that sufficient amounts of dopamine binding to D1 receptors in the PFC enhance cognitive functioning by optimally suppressing "noise" and enhancing "signals" to guide behavior (Vijayraghavan, Wang, Birnbaum, Williams, \& Arnsten, 2007). Too little or too much D1 receptor binding in the PFC impairs cognitive function (Zahrt, Taylor, Mathew, \& Arnsten, 1997). When D1 receptors are flooded by excess dopamine, oversuppression of both noise and signal occurs, impairing the PFC's ability to guide behavior (Vijayraghavan et al., 2007; Zahrt et al., 1997). In humans, treatment with a dopamine receptor agonist before performing working memory tasks in an fMRI scanner led to decreased activity in 
frontal regions and decreased performance relative to those who took placebo (Gibbs \& D'Esposito, 2005). Importantly, this suppression of neuronal activity in the PFC could impact the PFC's regulatory influence over subcortical structures, resulting in amplified emotional function and response (Arnsten \& Goldman-Rakic, 1998), which has implications for enhanced risktaking and other reward-motivated behavior.

In human adults, acute stress yields alterations in neural activation of mesolimbicprefrontal circuitry, including the striatum, insula, and prefrontal regions (Kogler et al., 2013; Pruessner et al., 2008), which may be due to the stress-related increases in dopamine release in ventral striatum and orbital frontal cortex observed in human positron emission tomography (PET) studies (Pruessner, Champagne, Meaney, \& Dagher, 2004). Indeed, this increased dopamine release is magnified in individuals who also evince increased cortisol levels in response to psychosocial stressors (Pruessner et al., 2004). In adults, stress leads to enhanced reward salience and greater reward-biased decisions coupled with decreased sensitivity to punishment during decision-making (Pabst, Brand, \& Wolf, 2013; Porcelli \& Delgado, 2009; Putman, Antypa, Crysovergi, \& van der Does, 2010; Starcke, Wolf, Markowitsch, \& Brand, 2008). For instance, stressed individuals who exhibited greater increases in cortisol chose options that led to potentially high reward but actual led to high punishment more often (Starcke et al., 2008). Another study reported that participants under acute stress took more risks in a loss domain versus gain domain (Porcelli \& Delgado, 2009), suggesting that stress may decrease sensitivity to losses. Therefore, it seems plausible that in adults, increases in acute stress-related cortisol lead to heightened activation in dopamine-rich mesolimbic regions in response to risk and reward, which then affects risky decision-making by increasing reward salience. 
Acute stress amplifies gender differences in risk-taking behavior and neural activation in regions implicated in risky decision-making; the directions of these effects, however, have been mixed. Lighthall, Mather, \& Gorlick, (2009) found that men under acute stress took more risks on the Balloon Analogue Risk Task (BART) compared to non-stressed men while women took less risks when stressed compared to non-stressed women. Moreover, while greater increases in stress-related cortisol was related to more conservative behaviors in women and not in men (Lighthall et al., 2009), another study found that greater stress-related cortisol increase in men led to decreased punishment-sensitive behavior and increased reward-sensitive behavior in situations that contain high potential rewards (Putman et al., 2010). In addition, Lighthall et al., (2012) found that in men, greater cortisol change was associated with enhanced dorsal striatum and anterior insula during decision-making on the BART, which led to greater reward collection and less risk taking, suggesting that men under stress tend to choose the low-risk option that resulted in more frequent gains of small rewards. The mixed findings in how stress affects risky decision-making in men and women could be due to differences in the decision-making tasks used across studies. In adolescents, it is less known how acute stress affects risky decisionmaking behavior. Galván and McGlennen (2012) used a within-subjects approach to investigate how daily stress influences risky decision-making on the Cups Task, a risky decision-making task, in adolescents (14-17 years) and emerging adults (18-21 years). Results indicated that all participants made more risky decisions during high relative to low stress. No developmental or gender differences were observed, which could be due to the younger age range of the "adults".

Surprisingly, the neural mechanisms by which acute stress affects risky decision-making in adolescents remain unexplored. Examining this question is important, given the increase in actual and perceived stress (Dahl \& Gunnar, 2009), greater inclination toward risk-taking 
compared to adults (Cauffman et al., 2010), enhanced engagement of mesolimbic circuitry in response to reward and risk (Braams, van Duijvenvoorde, Peper, \& Crone, 2015; Galván et al., 2006; Lamm et al., 2014) and heightened stress response that occurs during puberty and adolescence (Dahl \& Gunnar, 2009; Stroud et al., 2009). Juvenile rodents show increased dopamine levels in the striatum to rewarding events compared to adults (Andersen, Thompson, Krenzel, \& Teicher, 2002; Hajnal, Smith, \& Norgren, 2004). There are also increased dopamine D1 and D2 receptors in the striatum and NAcc in adolescence relative to adulthood (Andersen et al., 2002; Lidow \& Rakic, 1992; Seeman et al., 1987) as well as increased expression of D1 receptor in the PFC via NAcc projections (Brenhouse, Sonntag, \& Andersen, 2008). Based on the inverted U-shape pattern between D1 receptor binding in the PFC and cognitive function, it is plausible that in adolescents, stress-related dopamine release might flood an already saturated dopaminergic system to risk and reward. Excess D1 receptor binding in the adolescent PFC could lead to greater dampening of PFC function, further subverting maturing regulatory functions in adolescents. If it were the case that saturated D1 receptors in the PFC in response to stress suppresses regulatory control in adolescents, we would expect to see decreased activation of frontal regions as a function of stress when making risky decisions and increased risk-taking in adolescents relative to adults.

The current study extends our previous work (Galván \& McGlennen, 2012) to examine the physiological and neurobiological mechanisms by which acute stress affects risky decisionmaking in adolescents 15-17 years of age. This narrow age range was chosen to capture development during the late-pubertal to post-pubertal stage of adolescence when pubertal hormones are more stable because differences in hormonal changes in adolescents still undergoing puberty could potentially confound examination of hormonal responses to stress 
(Dahl \& Gunnar, 2009). In addition, instead of inducing stress in participants through a laboratory stressor, we chose to utilize a daily diary design to monitor adolescents' daily stress (Galván \& McGlennen, 2012) because it is uncertain whether or not traditional laboratory stressors (e.g., Trier Social Stress Test) are experienced similarly in adolescents and adults relative to how they would respond to their own developmentally appropriate daily stressors. Using a within-subjects design, participants came into the laboratory to complete measures on a day when they endorsed feeling high levels of stress and on another day when they endorsed feeling low levels of stress. During a brain scan, participants completed the Cups task (Levin \& Hart, 2003; Levin, Weller, Pederson, \& Harshman, 2007), which allows for differentiation of advantageous and disadvantageous risks to investigate how acute stress affects adaptive risktaking, which is more akin to decision-making in the real-world where some risks are advantageous while others are disadvantageous. Extant research in adolescents has not been aimed at uncovering whether behavioral and neural patterns differentiate between advantageous and disadvantageous risk taking. Instead, it has mainly been assumed that risk-taking in the lab translates to disadvantageous risk-taking in real life. The Cups task allowed us to investigate how stress affects the neural computations used to assess risky decision-making such as modifying risk propensity (risk-seeking vs. risk-aversion), sensitivity to gains and losses, and/or sensitivity to expected value (EV). To investigate developmental differences in stress effects, a comparison group of traditionally defined adults (25-30 years of age) was also tested.

We predicted that, compared to adults, adolescents would show heightened striatal activation and dampened PFC activation in response to risk-taking under high stress relative to low stress, which would lead to increased risky decision-making in adolescents relative to adults. We also predicted that these effects would be mediated by cortisol response to stress. We did not 
have specific predictions regarding gender differences in how stress affects risky decisionmaking in adults or adolescents, as previous findings have been mixed; therefore, gender was included as an exploratory variable.

\section{Method}

\subsection{Participants}

Twenty-two adolescents (14 females) between $15-17$ years of age $(M=16.55, S D=.67)$ and twenty-three adults (12 females) between 25-30 years of age $(M=27.27, S D=1.72)$ participated in the study. Participants were recruited through advertisements on the UCLA campus, surrounding neighborhoods, and local online classifieds and forums. Participants were excluded if they had any metal objects in their bodies (e.g., braces, permanent retainers), a diagnosis of a psychiatric or developmental disorder, claustrophobia, were left-handed, or were pregnant. Informed consent was obtained from all adult participants; parental consent and assent was obtained from all participants under the age of 18 in accordance with procedures approved by the UCLA Institutional Review Board. The Wechsler Abbreviated Scale of Intelligence (WASI) was administered to estimate IQ. Adolescents and adults did not significantly differ in IQ $(t(42)=-.868, p=.390)$ or ethnic composition $\left(X^{2}(5, \mathrm{~N}=44)=5.524, p=.355\right)$. Because cortisol levels are influenced by socioeconomic status (Chen, Cohen, \& Miller, 2010; Cohen et al., 2006; Desantis, Kuzawa, \& Adam, 2015) and recent stressful life events (Armbruster et al., 2012), analyses were conducted with these variables as covariates. Socioeconomic status (SES) was determined by participant's maternal level of education. Recent stressful life events (SLE) was determined by participant's self-reported number of stressful life experiences in the past 6 months on a Life Events checklist (e.g., death of close family member, begin new school, change in living conditions, change in sleep habits, breakup with significant other, etc.). Adolescents and 
adults did not significantly differ in $\operatorname{SES}\left(X^{2}(6, \mathrm{~N}=44)=6.210, p=.400\right)$, or in the number of recent SLE, $(t(42)=.411, p=.683)($ Table 1$)$.

Table 1. Demographics

\begin{tabular}{lcc}
\hline & Adolescents & Adults \\
\hline $\mathrm{N}$ & $22(14$ female) & $22(12$ female) \\
Age range (years) & $15.21-17.97$ & $25.48-30.81$ \\
Age (years), Mean (SD) & $16.55(.67)$ & $27.27(1.72)$ \\
IQ, Mean (SD) & $114.59(13.25)$ & $117.68(10.15)$ \\
Mother's Level of Education, N (\%) & \\
0 = Did not finish high school & $1(4.55 \%)$ & $0(0.00 \%)$ \\
$1=$ High School Diploma & $4(18.18 \%)$ & $6(26.09 \%)$ \\
$2=$ GED & $2(9.09 \%)$ & $0(0.00 \%)$ \\
$3=$ College: Associate Degree & $1(4.55 \%)$ & $2(9.09 \%)$ \\
$4=$ College: Bachelor Degree & $5(22.73 \%)$ & $9(40.91 \%)$ \\
5 = Master's Degree & $8(36.36 \%)$ & $4(18.18 \%)$ \\
6 = Other (e.g., professional degree) & $1(4.55 \%)$ & $1(4.55 \%)$ \\
Ethnicity, N (\%) & & \\
African American & $1(4.55 \%)$ & $1(4.55 \%)$ \\
Asian American & $1(4.55 \%)$ & $6(26.09 \%)$ \\
Caucasian & $10(45.46 \%)$ & $10(45.46 \%)$ \\
Hispanic/Latino & $5(22.73 \%)$ & $2(9.09 \%)$ \\
Multi-ethnic & $4(18.18 \%)$ & $2(9.09 \%)$ \\
Other (e.g., Native American) & $1(4.55 \%)$ & $1(4.55 \%)$ \\
Recent Stressful Life Events (Number), Mean (SD) & $3.59(2.61)$ & $3.23(3.22)$ \\
Low Stress Rating, Mean (SD) & $2.091(1.065)$ & $2.545(1.057)$ \\
High Stress Rating, Mean (SD) & $4.318(1.287)$ & $4.636(1.56)$
\end{tabular}

\subsection{Procedure}

Each participant was enrolled in the study for two weeks (Figure 1). Participants were invited to come to the laboratory for an initial intake. After providing consent, participants completed a short battery of questionnaires and received instructions regarding the study procedures. Participants provided salivary cortisol samples at the beginning and end of the intake 
visit to assess baseline physiological stress response to the novel, laboratory setting. Salivary cortisol is a commonly used indicator of an organism's response to stress (Hellhammer, Wüst, \& Kudielka, 2009).

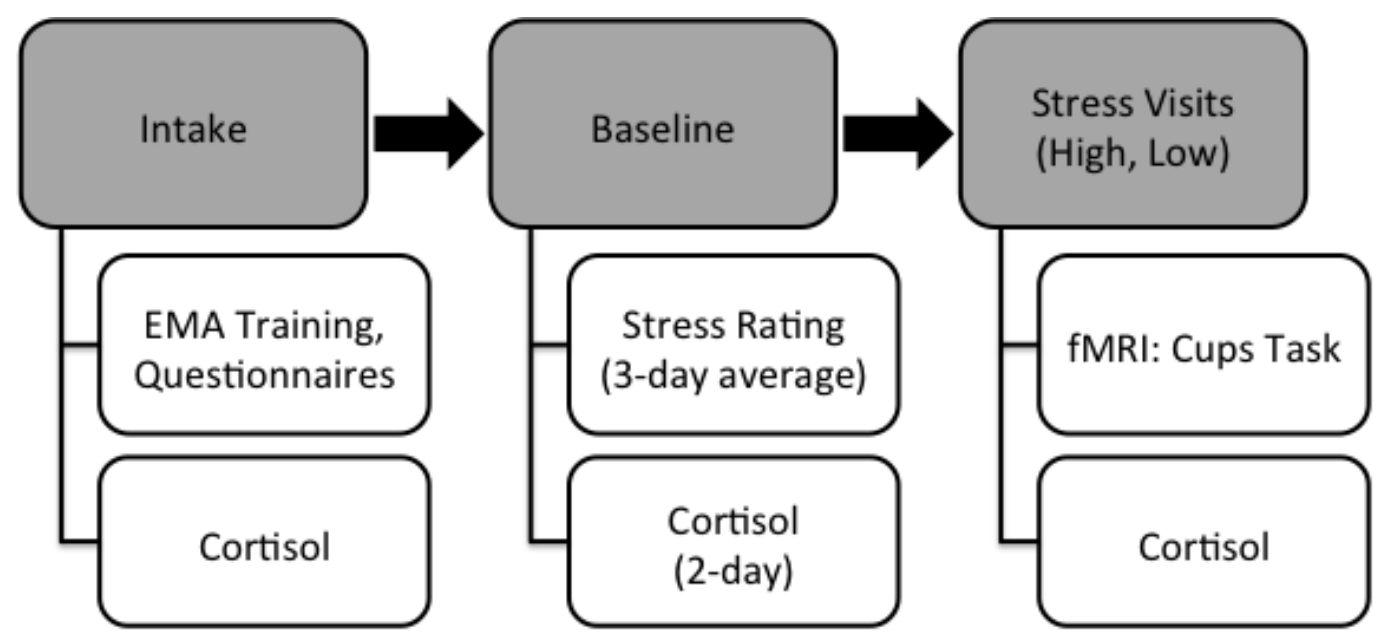

14 days

Figure 1. Study design.

\subsection{Daily Stress Assessment}

Daily stress was assessed using an ecological momentary assessment (EMA; Bolger, Davis, \& Rafaeli, 2003) method, a procedure in which participants were contacted daily through smartphones and reported daily ratings of subjective feelings of stress. A smartphone was available from the lab to participants who did not have a cellular phone and/or a text-messaging plan. However, all participants in the study used their own personal cellular phones. The EMA method has been shown to be successful at capturing naturally occurring stress (Almeida, McGonagle, \& King, 2009; Galván \& McGlennen, 2012; Rahdar \& Galván, 2014). In the current study, participants were unsystematically contacted three times per day between the hours of 


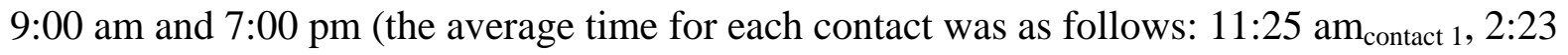

$\mathrm{pm}_{\text {contact2 }}, 5: 00 \mathrm{pm}_{\text {contact } 3}$ ) over the span of 2 weeks via text messages and were asked to indicate the level of stress that they experienced in the last hour using a Likert scale $(1=$ not stressed; $7=$ very stressed).

\subsection{Baseline Assessment}

During the first three days, a baseline composite stress rating for each participant was determined by averaging the stress ratings provided via text messages throughout the day across the three days (average baseline stress rating $=3.0211, S D=.904$ ). Concurrently, during the first two consecutive days, participants collected salivary cortisol via passive drool at home to evaluate each participant's baseline diurnal cortisol pattern. Participants were instructed to collect $1 \mathrm{~mL}$ of saliva at awakening, 30 minutes post-awakening, at 4:30 pm, and at 8:30 pm (participants noted the exact time of collections) using Salivette® test tubes (Sarstedt, Germany). This method and timing of salivary cortisol collection has been shown to be standard and reliable (Clements, 2012). All samples were stored at $-20^{\circ} \mathrm{C}$ in a laboratory freezer at UCLA until time of shipment. Salivary cortisol analyses were conducted as described by Strahler, Mueller, Rosenloecher, Kirschbaum, \& Rohleder (2010).

Two subsequent laboratory visits were categorized into high-stress and low-stress visits, counterbalanced across subjects, as based on stress ratings relative to baseline stress ratings. High-stress state was determined when participants endorsed stress ratings that were at least one standard deviation above their baseline stress rating. If the baseline stress rating was already relatively high, the threshold was then a 1-point increase in stress rating. Similarly, low-stress state was determined when participants endorsed stress ratings that were at least one standard 
deviation below their baseline stress rating. If baseline stress rating was already relatively low, the threshold was then a 1-point decrease in stress rating.

\subsection{Stress Visits}

After a high-stress state or low-stress state was determined, participants were asked to visit the laboratory in the late afternoon of the same day. At each stress visit, upon arrival, participants were asked to rate the level of stress $(1=$ not stressed; $7=$ very stressed $)$ that they

experienced prior to coming into the laboratory across a number of stressors (e.g., conflict with roommates or parents, family demands, academic stress, peer stress, job/other responsibility stress, relationship stress); participants were given the option to list their own stressor if a stressor was not included in the list. For each stress visit, salivary cortisol was collected before participants entered the functional magnetic resonance imaging (fMRI) scanner to assess physiological response to stress as close in time to stressor onset as possible and once again after the fMRI scan to assess stress reactivity to the novel brain-scanning environment. Duration between reported stress (stressor) and brain scan ranged from 30 minutes to 5 hours and 30 minutes $(M=2$ hours and 7 minutes, $S D=1$ hour, 6 minutes $)$. Duration did not significantly differ by stress state $(p=.263)$, or age group $(p=.729)$. During the visit, participants underwent an fMRI scan while performing the Cups Task, a risky decision-making task (Figure 2). 


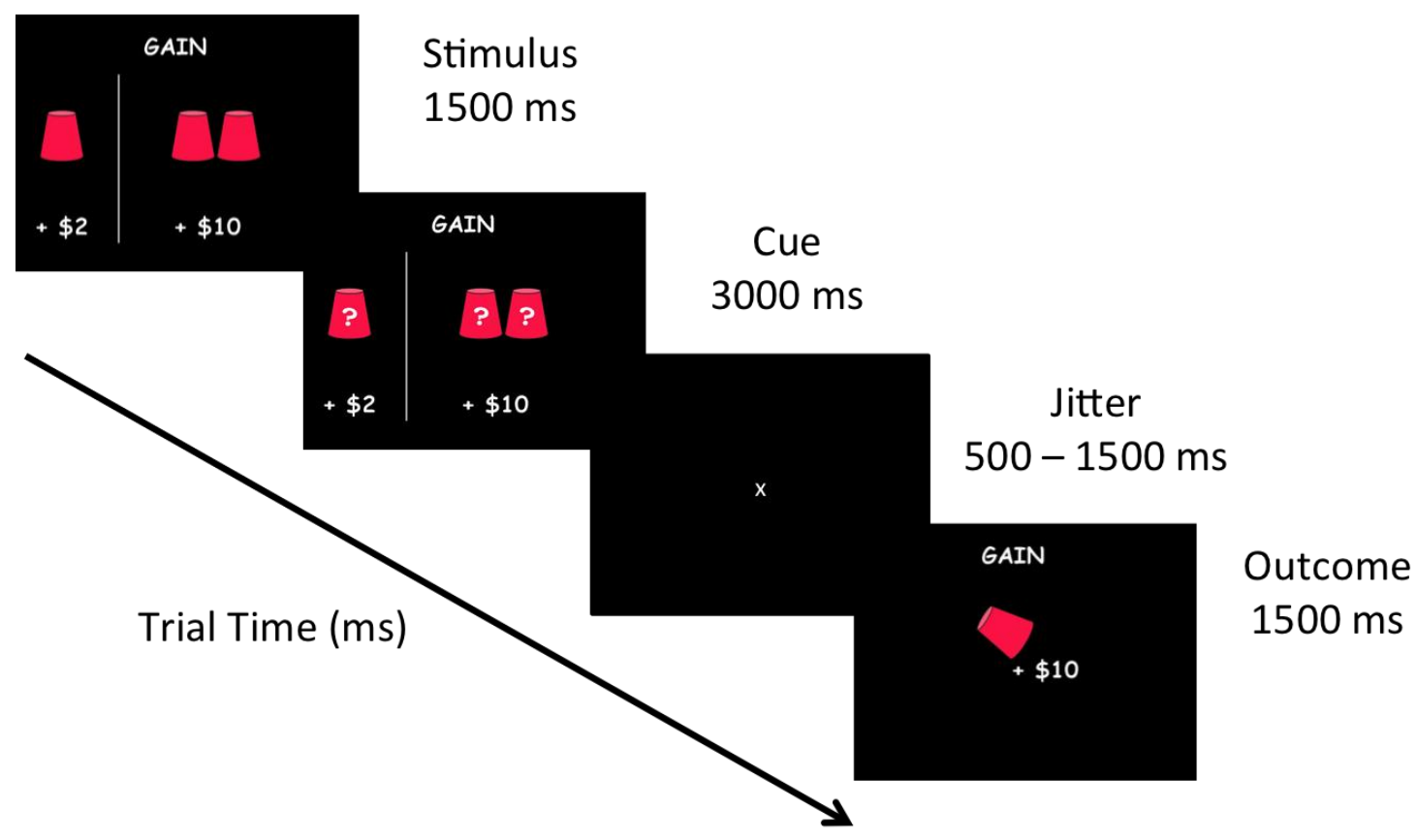

Figure 2. Schematic of Cups Task. Each stimulus was presented for 1500 ms. After stimulus presentation (Stimulus), participants are asked to choose between the certain (left) side or the uncertain (right) side (Cue). Once participants made their decision, a jittered inter-stimulusinterval (ISI) was shown for 500-1500ms followed by outcome presentation (Outcome) for 1500 ms.

\subsection{Risky Decision-Making Task}

The Cups Task (Levin \& Hart, 2003; Levin et al., 2007) measures decision-making under uncertainty, as individuals often make decisions under uncertain and stressful conditions. The Cups Task has been used in previous fMRI studies to study the neural correlates of risky decision-making (Xue et al., 2009; Xue, Lu, Levin, \& Bechara, 2013). Participants were presented with two task frames (Frame): a frame where they had the opportunity to gain money (Gain Frame, $n=54$ trials), and one where they could lose money (Lose Frame, $n=54$ trials). Depending on the frame, participants were asked to choose between a certain gain (or loss) and 
an uncertain gain (or loss). The neutral terms "certain" and "uncertain" were used during explanation of the task to participants to prevent suggestion of any connotations or biases that might be associated with the terms "safe" and "risky," which are used throughout the report. The certain option was to win (or lose) $\$ 2$, while the uncertain option led to a probability ( $20 \%, 33 \%$, or $50 \%$ ) of either a larger win (or loss) $(\$ 4, \$ 6$, or $\$ 10)$ or no win (or loss). The certain option consisted of one flipped-over cup with $\$ 2$ written underneath it, indicating to participants that there is a $100 \%$ chance of gaining (or losing) $\$ 2$. In the uncertain option, the number of cups varied between two (50\%), three (33\%), and five (20\%) cups, and the amount indicated underneath each set of cups varied between $\$ 4, \$ 6$, and $\$ 10$. There were three trial types $(n=$ 36/trial type) that differed in expected value (EV = value $\mathrm{x}$ probability). The advantageous EV trials (ADV), in which the EV was greater than $\$ 2$ in the gain frame or less than $\$ 2$ in the lose frame, consisted of the 50\%-\$6,50\%-\$10, and 33\%-\$10 trials in the gain frame and $33 \%-\$ 4$, $20 \%-\$ 4$, and $20 \%-\$ 6$ trials in the lose frame; in these trials, the uncertain choice yielded better EV than the certain choice. The disadvantageous EV trials (DIS), in which the EV was less than $\$ 2$ in the gain frame and greater than $\$ 2$ in the lose frame, consisted of the $33 \%-\$ 4,20 \%-\$ 4$, and $20 \%-\$ 6$ trials in the gain frame and the 50\%-\$6,50\%-\$10, and 33\%-\$10 trials in the lose frame; in these trials, the uncertain choice yielded worse EV than the certain choice. In the equal EV trials (EQEV), in which the EV was $\$ 2$, consisted of the $50 \%-\$ 4,33 \%-\$ 6$, and $20 \%-\$ 10$ trials in both gain and lose frames; in these trials, the uncertain choice yielded the same EV as the certain choice. Participants were instructed to consider each choice carefully and to earn as much money as possible in the game because at the end of the game, the computer would randomly select an outcome based on their decisions and that outcome would be added to or subtracted from their study compensation. Participants did not actually lose any money; this information was not 
disclosed to participants until after completion of the task. Thus, participants could earn between $\$ 2$ and $\$ 10$ in addition to study compensation. All participants completed two 7 -minute runs $(\mathrm{n}=$ 108 total trials) of the task.

\subsection{MRI Data Acquisition}

Functional imaging data were collected on a 3 Tesla Siemens Trio MRI scanner using a gradient-echo, echo-planar image (EPI) sequence (TR: 2 s, TE: $30 \mathrm{~ms}$, flip angle: $90^{\circ}, 271$ volumes, 34 slices, slice thickness: $4 \mathrm{~mm}$ ). A T2-weighted, matched bandwidth (MBW), highresolution anatomical scan and magnetization-prepared rapid-acquisition gradient echo (MPRAGE) scan were acquired for registration purposes (TR: 2.3, TE: 2.1, FOV: 256, matrix: 192 x 192, sagittal plane, slice thickness: $1 \mathrm{~mm}, 160$ slices). The orientation for the MBW and EPI scans was oblique axial to maximize brain coverage. E-prime software was used for task presentation.

\subsection{Image Preprocessing and Registration}

Preprocessing and statistical analyses were performed using FMRIB's Software Library (FSL) 5.0.9 (www.fmrib.ox.ac.uk/fsl). Preprocessing included motion correction, non-brain matter removal using FSL brain extraction tool (BET), spatial smoothing (5mm FWHM Gaussian kernel) to increase the signal-to-noise ratio, and filtering in the temporal domain using a nonlinear high-pass filter (100-s cutoff). All data reported are from scans with $\leq 2 \mathrm{~mm}$ in translational movement. EPI images were registered to the MBW scan, then to the MPRAGE scan, and finally into standard the Montreal Neurological Institute (MNI) space (MNI152, T1

2mm) using linear registration with FSL FMRIB's Linear Image Registration Tool (FLIRT).

\subsection{Functional Neuroimaging Data Analysis}


A general linear model (GLM) with multiple regressors for each event type (decisionmaking and outcome) was used for fMRI analyses. Statistical modeling was first performed for each image, and variables of interest were compared between groups using two-sample $t$ tests. Regressors of interest were created by convolving a delta function representing the onset time of each event with a canonical (double gamma) HRF. Onset time for decision-making event was defined as the trial onset for the stimulus presentation. Onset time for outcome event was defined as the trial onset for the outcome presentation. To characterize the effect of EV on risky decision-making, a GLM with multiple regressors was used for fMRI analyses. For each participant, each stress visit, and each run, eight explanatory variables were modeled: 1) risky choice in ADV trials; 2) risky choice in DIS trials; 3) risky choice in EQEV trials; 4) safe (nonrisky) choice in ADV trials; 5) safe choice in DIS trials; 6) safe choice in EQEV trials; 7) outcome presentation; and 8) missed/invalid trials. Six motion parameters were modeled as events of no interest. Temporal derivatives were included as coviariates of no interest. Inter-trial and inter-stimulus interval fixation points were not explicitly modeled and therefore served as an implicit baseline.

fMRI analyses focused on participant choice (risky or safe) and EV condition (ADV, DIS, EQEV) for high stress scans and low stress scans. To examine the effects of EV on risky decision-making, the following contrasts were modeled for each participant and stress visit: 1) ADV Risk > ADV Safe; 2) DIS Risk > DIS Safe; 3) EQEV Risk > EQEV Safe; 4) ADV Risk > DIS Risk; 5) DIS Risk > ADV Risk; 6) ADV Risk > EQEV Risk; 7) EQEV Risk > ADV Risk; 8) EQEV Risk > DIS Risk; and 9) DIS Risk > EQEV Risk. A fixed effects voxel-wise analysis combined each of two runs for each stress visit for each participant at the second level. 
2 (Stress) x 2 (Age) x 2 (Gender) repeated measures analyses of variance (ANOVA) were conducted at the group level using the FMRIB local analysis of mixed effects (FLAME1) module in FSL. Z (Gaussianized T) statistic images were threshold using clusters determined by $\mathrm{Z}>2.3$ and a whole-brain corrected cluster significant threshold of $\mathrm{p}<.05$ using Gaussian Random Field theory. Tests were corrected for family-wise errors (FWE). Anatomical localization within each cluster was obtained by searching within maximum likelihood regions from the FSL Harvard-Oxford probabilistic atlas. Regions of activation for all contrasts are listed in Table 4.

\subsection{Statistical Analysis of Behavioral \& Cortisol Data}

The percentage of risks taken on the Cups Task for each EV type was calculated by counting the number of times participants chose the risky option of each EV type divided by the total number of trials presented of each EV type, multiplied by 100 .

Total earnings on how much participants would have won on the Cups task based on participants' choices and outcomes were calculated for each stress visit.

Since the time of day that participants collected salivary cortisol for baseline varied between participants, a series of linear regressions were used to individually model each participant's baseline diurnal pattern of cortisol by regressing cortisol values (Cortisol) on the time of day the cortisol sample was collected (Time) and the quadratic form of time of day $\left(\right.$ Time $\left.^{2}\right)$ across the two days of collection: Cortisol $=b_{0}+b_{1}($ Time $)+b_{2}\left(\right.$ Time $\left.^{2}\right)+$ e. One limitation in having stress states determined by participants is that the time of day that participants visited the laboratory varied. As a consequence, the time of day the salivary cortisol was collected by participants on the laboratory visits varied. One way to account for this variability in time was to first interpolate the predicted cortisol value for the time of day of the 
visit by substituting the time of day (in hours) into the Time and Time ${ }^{2}$ variables in each participant's predicted regression equation. This provided a predicted baseline cortisol value for a particular time of day for a particular participant. This predicted cortisol value was then used to calculate a difference score from the actual cortisol value collected during each laboratory visit. Three difference scores in cortisol were calculated for the three laboratory visits for each participant: Lab Cortisol Difference, High Stress Cortisol Difference, and Low Stress Cortisol Difference. These difference scores were used as a measure of cortisol reactivity to stress.

\section{Results}

\subsection{Stress Ratings}

A 2 (Stress) x 2 (Age) x 2 (Gender) repeated measures ANOVA on stress ratings revealed a main effect of stress condition, $F(1,40)=73.592, p<.001$ : stress ratings under high stress $(M=4.48, S D=1.42)$ were greater than stress ratings under low stress $(M=2.32, S D=$ 1.07). No effect of age, gender, age $x$ gender interaction was found on stress ratings. The effect of stress condition on stress ratings (i.e., difference in stress ratings between high stress and low stress) did not differ by age, gender or age x gender (Figure 3A). There were no significant associations between stress ratings and behavioral performance, cortisol, or brain activation.

\subsection{Salivary Cortisol}

A 2 (Stress) x 2 (Age) x 2 (Gender) repeated measures analysis of covariance (ANCOVA) on cortisol difference, controlling for cortisol difference during intake (Lab Cortisol Difference), SES, and recent SLE revealed a main effect of stress, $F(1,32)=6.677, p=.038$ : cortisol difference from baseline during high stress $(M=3.10 \mathrm{nmol} / \mathrm{L}, S D=8.42)$ was greater than cortisol difference from baseline during low stress $(M=-.319 \mathrm{nmol} / \mathrm{L}, S D=7.86)$. This effect confirmed that participants were indeed experiencing greater levels of stress during high 
stress relative to low stress. No effect of age, gender, age x gender on cortisol difference was found. The effect of stress on cortisol difference also did not differ by age, gender, or age $\mathrm{x}$ gender (Figure 3B). There were no significant associations between cortisol and brain activation.

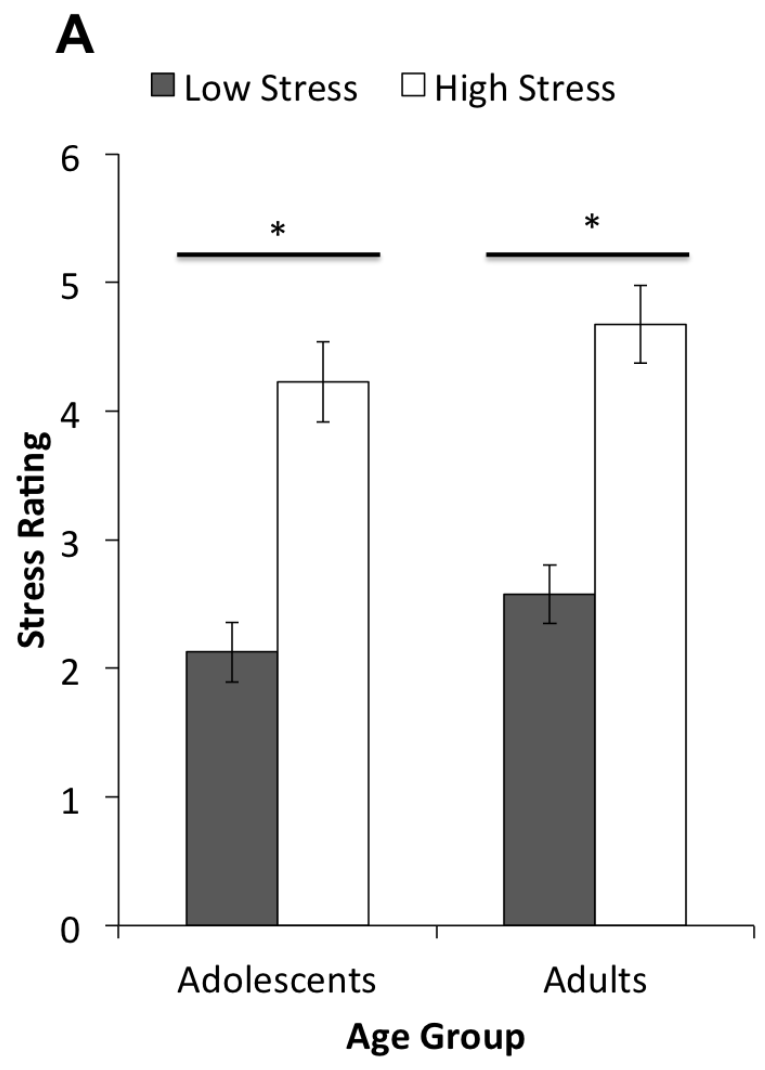

\section{B}

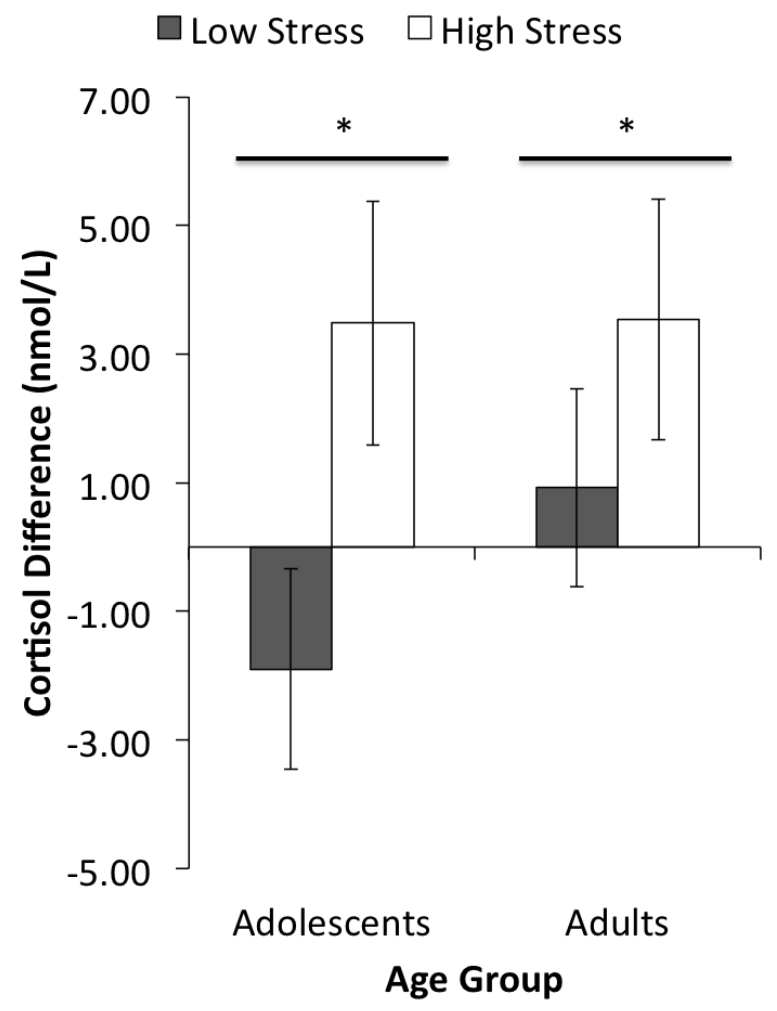

Figure 3. (A) Stress ratings and (B) cortisol response on high stress days were significantly higher than stress ratings and cortisol response on low stress days. This effect of stress on ratings and cortisol response did not differ by age group or gender. Errors bars denote 1 standard error.

\subsection{Risky Behavior}

A 2 (Stress) x $3(\mathrm{EV})$ x 2 (Frame) x 2 (Age) x 2 (Gender) repeated-measures analysis of covariance (ANCOVA) on percentage of risky decisions controlling for SES and SLE revealed a 
main effect of EV, $F(2,37)=14.755, p<.001$, a Stress $x \mathrm{EV}$ interaction, $F(2,37)=3.737, p=$ .033 , a Stress $x$ EV x Gender interaction, $F(2,37)=4.838, p=.014$, and a Stress $x$ EV x Age x Gender interaction, $F(2,37)=3.251, p=.05$. There were no significant effects of Frame $(p>$ $.05)$; therefore, gain and loss trials were collapsed across each level of EV for analyses.

Descriptive statistics of behavioral data are listed in Table 2.

Table 2.Behavioral data

\begin{tabular}{lccccccccc}
\multicolumn{4}{c}{ Adolescents } & \multicolumn{2}{c}{ Adults } & \multicolumn{2}{c}{ Adolescents } & \multicolumn{2}{c}{ Adults } \\
& \multicolumn{2}{c}{ M (SD) } & \multicolumn{2}{c}{ M (SD) } & \multicolumn{2}{c}{ M (SD) } & & M (SD) \\
& Male & Female & Male & Female & Male & Female & Male & Female \\
\hline ADV risks (\%) & $91.67(8.91)$ & $81.35(12.87)$ & $92.22(12.41)$ & $80.32(17.26)$ & $81.94(18.49)$ & $78.37(12.04)$ & $91.11(8.05)$ & $82.18(15.96)$ \\
Gain & $93.75(8.63)$ & $89.68(9.95)$ & $94.44(12.83)$ & $85.65(10.18)$ & $91.67(6.64)$ & $85.32(15.04)$ & $93.33(11.65)$ & $86.11(14.31)$ \\
Lose & $89.58(12.04)$ & $73.02(21.22)$ & $90.00(12.78)$ & $75.00(27.16)$ & $72.22(31.00)$ & $71.43(18.98)$ & $88.89(8.28)$ & $78.24(22.65)$ \\
DIS risks (\%) & $25.00(20.52)$ & $25.60(20.04)$ & $7.22(11.43)$ & $24.31(17.45)$ & $29.86(27.29)$ & $27.58(20.25)$ & $13.89(18.05)$ & $28.94(18.82)$ \\
Gain & $30.56(26.73)$ & $27.78(19.00)$ & $11.11(17.57)$ & $28.70(29.00)$ & $33.33(27.38)$ & $32.14(25.05)$ & $17.78(20.75)$ & $32.87(19.88)$ \\
Lose & $19.44(17.82)$ & $23.41(24.77)$ & $3.33(7.03)$ & $19.91(20.16)$ & $26.39(34.60)$ & $23.02(21.33)$ & $10.00(16.31)$ & $25.00(20.58)$ \\
EQEV risks (\%) & $60.42(22.80)$ & $54.37(16.00)$ & $41.39(18.91)$ & $56.02(22.93)$ & $56.25(21.55)$ & $52.18(14.13)$ & $54.44(26.52)$ & $55.79(23.31)$ \\
Gain & $67.36(29.91)$ & $62.30(19.02)$ & $48.89(23.83)$ & $54.17(26.39)$ & $65.28(19.19)$ & $60.71(24.41)$ & $59.44(29.98)$ & $58.80(27.26)$ \\
Lose & $53.47(29.39)$ & $46.43(21.97)$ & $33.89(18.78)$ & $57.87(27.06)$ & $47.22(34.63)$ & $43.65(17.82)$ & $49.44(30.70)$ & $52.78(25.24)$ \\
\hline
\end{tabular}

$\mathrm{ADV}=$ advantageous $; \mathrm{DIS}=$ disadvantageous $; \mathrm{EQEV}=$ equal expected value; $\mathrm{M}=$ mean; $\mathrm{SD}=$ standard deviation

To characterize the nature of the Stress x EV x Age x Gender interaction, developmental differences in how stress affects risky decision-making (i.e., Stress x EV x Age interaction) were analyzed between males and females. Results revealed a Stress x EV x Age interaction in males, $F(2,13)=4.764, p=.028$, but not in females $(p>.05)$, indicating that stress influenced risky decision-making similarly in adolescent and adult females (Figure 4A). Post-hoc simple effects were conducted to describe the nature of the Stress x EV x Age interaction in males. Under low stress, adult males and adolescent males took more ADV risks than both DIS risks and EQEV risks, and also more EQEV risks than DIS risks, $F(2,37)=48.925, p<.001$ and $F(2,37)=$ 18.041, $p<.001$, respectively, confirming that participants were sensitive to EV. However, the 
difference between ADV risks and DIS risks in adult males was greater than that in adolescent males under low stress, $F(1,38)=4.798, p=.035$. Similar patterns of $\mathrm{EV}$ were also found under high stress; however, developmental differences in risk-taking by EV emerged. For adolescent males, there was an increase in ADV risks taken under high relative to low stress, $F(1.38)=$ $6.955, p=.012$, and no stress-related changes in DIS risks or EQEV risks, resulting in a greater difference between ADV risks and DIS risks taken under high stress compared to that under low stress, $F(1,38)=6.807, p=.013$ (Figure 4B). In contrast, among adult males, there was a decrease in EQEV risks taken under high stress relative to low stress, $F(1,38)=8.981, p=.005$, and no stress-related changes in $\mathrm{ADV}$ risks or EQEV risks, resulting in a greater difference between $\mathrm{ADV}$ risks and $\mathrm{EQEV}$ risks taken under high stress compared to that under low stress, $F(1,38)=15.137, p<.0001$ (Figure 4B). Moreover, as can be observed in Figure 4B, though the difference (slope) between DIS and EQEV risks did not differ between adolescent males and adult males, adolescent males took more DIS risks relative to adult males under high stress $(F(1$, $38)=5.302, p=.027)$, evidence of an increased risk preference in adolescent males versus adult males for choices that were not advantageous despite successful differentiation of EV. 


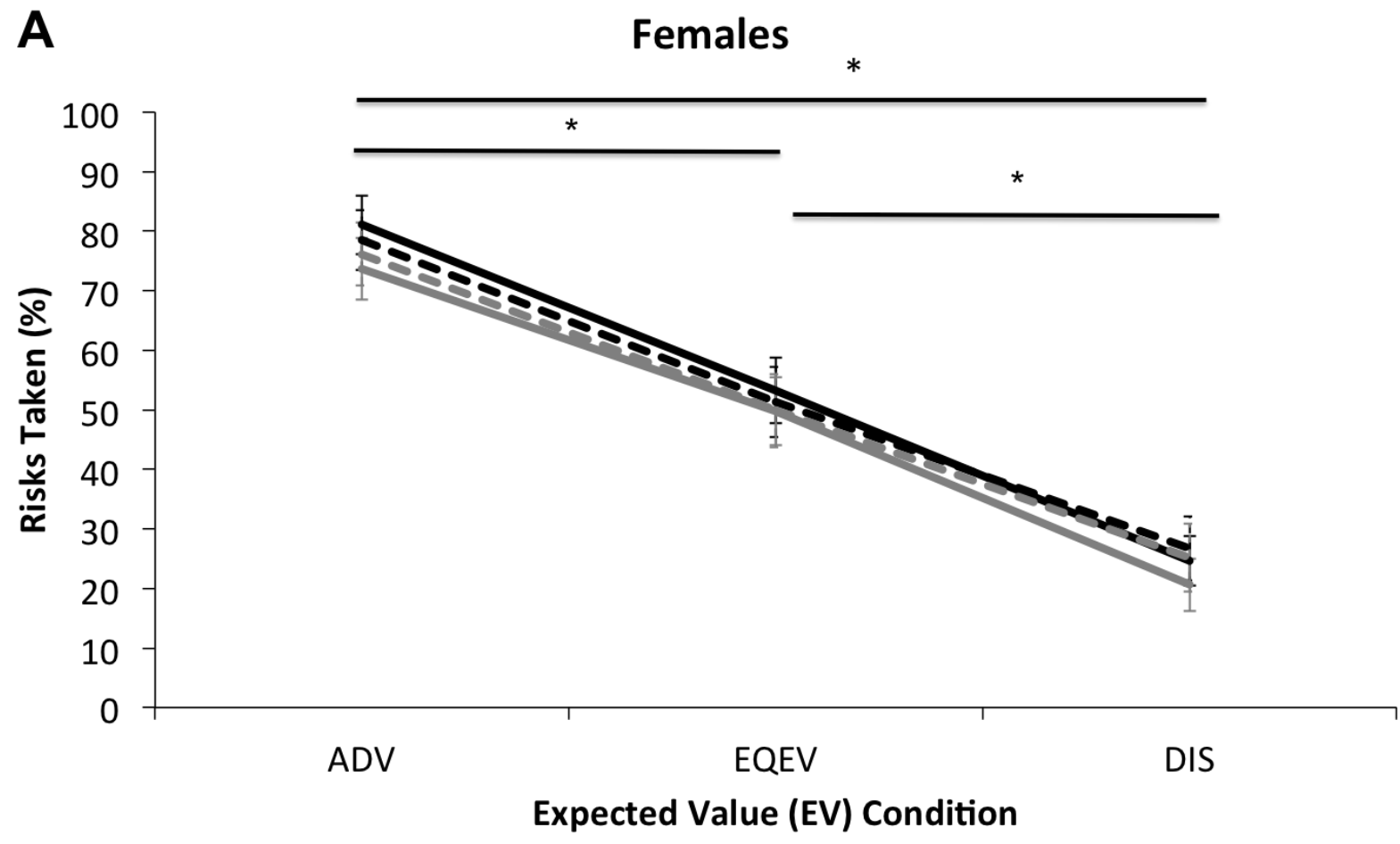

-Adolescent Female: High Stress - - Adolescent Female: Low Stress

-Adult Female: High Stress _ - - Adult Female: Low Stress 


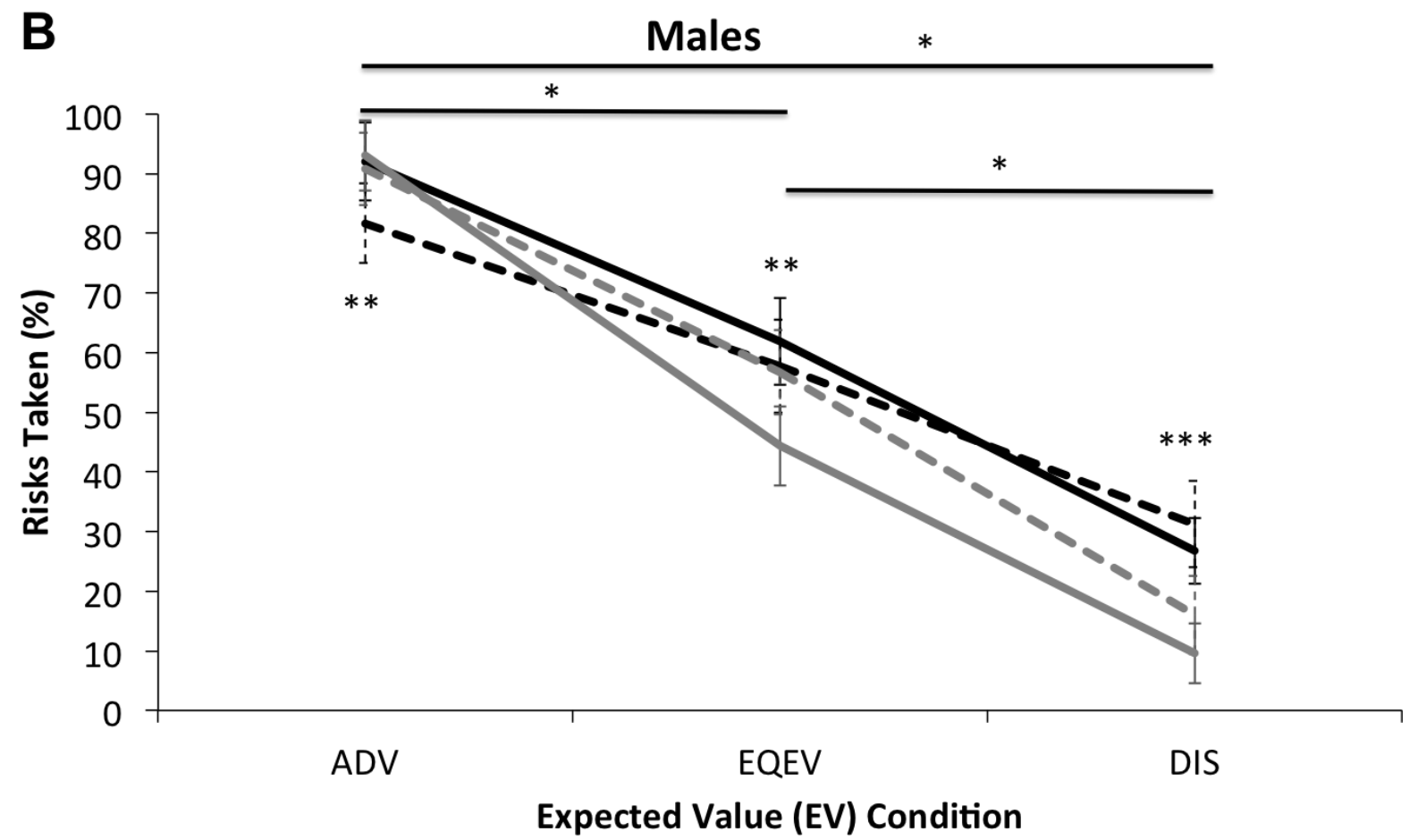

-Adolescent Male: High Stress - - Adolescent Male: Low Stress

-Adult Male: High Stress - - Adult Male: Low Stress

Figure 4. (A) There was a main effect of expected value (EV) in females such that more advantageous (ADV) risks were taken than risks of equal expected value (EQEV)*, which were greater than disadvantageous (DIS) risks*, $F(2,37)=93.904, p<.001$. (B) Males also showed significant effects of EV that was modified by stress and age (Stress $x$ EV x Age interaction) such that adolescent males took more ADV risks under high stress relative to low stress** while adult males took fewer EQEV risks under high stress relative to low stress**. Under high stress, adolescent males took more DIS risks than adult males***. Error bars denote 1 standard error.

Alternatively, given a 4-way interaction, developmental differences in how stress affects gender differences in risk-taking were also analyzed. Analyses comparing the Stress x EV x Gender interaction between adolescents and adults found that the Stress x EV x Gender 
interaction was significant only for adults, $F(2,17)=4.883, p=.021$, and not for adolescents $(p$ $=.374$ ), which indicate that gender differences in how stress affects risky decision-making may be stronger in adults than in adolescents. The Stress $x$ EV x Gender interaction in adults was driven by stress-related changes in utilization of EV information to guide risk-taking in adult males (described above) while adult females showed no significant effects of stress.

Average total earnings on the Cups task for each group as a function of stress is displayed in Table 3. A 2 (Stress) x 2 (Age) x 2 (Gender) repeated measured ANCOVA on earnings revealed no significant effects.

\begin{tabular}{cccc}
\hline Males & $\begin{array}{c}\text { Low Stress } \\
\text { Earnings }\end{array}$ & $\begin{array}{c}\text { High Stress } \\
\text { Earnings }\end{array}$ & within-subjects effect \\
\hline Adolescent & $\$ 12.62(38.70)$ & $\$ 29.31(38.50)$ & $p=0.26$ \\
Adult & $\$ 37.79(33.50)$ & $\$ 29.25(51.25)$ & $p=0.338$ \\
between-subjects effect & $p=0.108$ & $p=0.997$ & \\
\hline \multicolumn{4}{c}{ High Stress } \\
Females & Low Stress & Earnings & within-subjects effect \\
\hline Adolescent & Earnings & $\$ 18.97(21.63)$ & $p=0.517$ \\
Adults & $\$ 8.27(29.26)$ & $\$ 31.51(28.90)$ & $p=0.074$ \\
\hline between-subjects effect & $\$ 9.66(30.07)$ & $p=0.369$ &
\end{tabular}

Table 3. Average total earnings on Cups Task as a function of stress. Standard deviations are reported in parentheses.

\subsection{Salivary Cortisol \& Risky Behavior}

EV differences were regressed on cortisol difference under high stress, controlling for age, gender, age $\mathrm{x}$ gender, SES, and SLE. Results revealed that as cortisol difference increases, the slope between ADV and EQEV risks gets flatter (difference decreases), $t(33)=-2.471, p=$ $.019, B=-.787$ while the slope between EQEV and DIS risks gets steeper (difference increases), $t(33)=2.381, p=.023, B=.777$. These effects are driven by increasing EQEV risks under high 
stress as cortisol difference increases, $t(33)=2.915, p=.006, B=1.052$. Cortisol difference was not associated with ADV risks or DIS risks under high stress.

\section{5 fMRI Data: Effects of EV and Stress on Risky Choices}

\subsubsection{Advantageous risks versus disadvantageous risks}

Within-subjects comparison of stress revealed a Stress x Age x Gender interaction in the right frontal pole ( $x=38, y=54, z=4, Z=3.63$ ) for ADV Risk > DIS Risk contrast (Figure 5A). Post-hoc analyses revealed that adolescent males showed a decrease in right frontal pole activation from low stress to high stress when taking ADV Risk > DIS Risk, $F(1,38)=4.727, p$ $=.036$; there was a trend showing increased right frontal pole activation from low stress to high stress when taking ADV Risk > DIS Risk for adult males, $F(1,38)=3.299, p=.077$ (Figure 5B). There were also Stress $x$ Gender interactions in the left caudate $(x=-12, y=14, z=10, Z=$ 3.44), and left inferior frontal gyrus (IFG), pars opercularis $(x=-54, y=12, z=20, \mathrm{Z}=3.5)$ for ADV Risk > DIS Risk contrast. Parameter estimates were extracted from these regions using a $6 \mathrm{~mm}$ sphere for analyses. Post-hoc analyses revealed that for females, averaging across age, there was an increase in caudate activation from low stress to high stress for ADV Risk > DIS Risk contrast $(F(1,38)=7.941, p=.008)$ with no significant effect of stress in left IFG $(p=$ .100). For males, averaging across age, there was a decrease in IFG activation from low stress to high stress for ADV Risk > DIS Risk contrast $(F(1,38)=10.144, p=.003)$ with no significant effects of stress in the left caudate $(p=.166)$. The main effects of ADV Risk $>$ DIS Risk contrast are listed in Table 4 and displayed in Figure 6. No significant regions of activation were found for the reverse contrast (DIS Risk > ADV Risk).

\section{Table 4. Significant regions of activation}

\begin{tabular}{lllllll}
\hline Contrast & Anatomical region & Hemisphere & $\mathrm{x}$ & $\mathrm{y}$ & $\mathrm{z}$ & Z-max \\
\hline
\end{tabular}




\section{ADV Risk > DIS Risk}

Mean Activation
Lateral occipital cortex

Frontal pole

Middle frontal gyrus

Precentral gyrus

Frontal pole

Anterior insula

Middle frontal gyrus

Putamen

Main effect of

Gender (Male >

Female)

\begin{tabular}{|c|c|c|c|c|c|c|}
\hline & Supramarginal gyrus & $\mathrm{L}$ & -54 & -30 & 50 & 2.8 \\
\hline & Anterior cingulate cortex & Medial & 2 & 10 & 42 & 2.75 \\
\hline & Paracingulate gyrus & Medial & -8 & 8 & 4 & 2.65 \\
\hline & Frontal pole & $\mathrm{L}$ & -36 & 38 & 34 & 2.63 \\
\hline & Middle frontal gyrus & $\mathrm{L}$ & -40 & 38 & 32 & 2.63 \\
\hline & Supramarginal gyrus & $\mathrm{R}$ & 48 & -40 & 48 & 2.5 \\
\hline \multirow[t]{9}{*}{ Stress x Gender } & Lateral occipital cortex & $\mathrm{L}$ & -48 & -74 & 28 & 3.81 \\
\hline & Lateral occipital cortex & $\mathrm{R}$ & 50 & -70 & 32 & 3.69 \\
\hline & Putamen & $\mathrm{L}$ & -20 & 10 & -8 & 3.53 \\
\hline & Inferior frontal gyrus, pars opercularis & $\mathrm{L}$ & -54 & 12 & 20 & 3.5 \\
\hline & Middle temporal gyrus & $\mathrm{R}$ & 64 & -8 & -20 & 3.5 \\
\hline & Caudate & $\mathrm{L}$ & -12 & 14 & 10 & 3.44 \\
\hline & Middle frontal gyrus & $\mathrm{L}$ & -34 & 22 & 46 & 3.4 \\
\hline & Frontal pole & $\mathrm{R}$ & 6 & 56 & -2 & 3.24 \\
\hline & Medial frontal cortex & Medial & -2 & 54 & -20 & 2.88 \\
\hline $\begin{array}{l}\text { Stress x Age } x \\
\text { Gender }\end{array}$ & Frontal pole & $\mathrm{R}$ & 38 & 54 & 4 & 3.63 \\
\hline \multicolumn{7}{|c|}{$A D V$ Risk $>$ EQEV Risk } \\
\hline \multirow[t]{12}{*}{ Mean activation } & Occipital pole & $\mathrm{L}$ & 18 & -96 & 16 & 3.11 \\
\hline & Middle frontal gyrus & $\mathrm{L}$ & -48 & 34 & 26 & 2.8 \\
\hline & Paracingulate gyrus & Medial & -2 & 8 & 44 & 2.77 \\
\hline & Anterior cingulate cortex & Medial & -4 & 12 & 38 & 2.68 \\
\hline & Frontal pole & $\mathrm{R}$ & 48 & 38 & 22 & 2.67 \\
\hline & Precentral gyrus & $\mathrm{L}$ & -50 & 6 & 20 & 2.65 \\
\hline & Middle frontal gyrus & $\mathrm{R}$ & 40 & 34 & 40 & 2.65 \\
\hline & Frontal pole & $\mathrm{L}$ & -38 & 56 & 10 & 2.63 \\
\hline & Anterior insula & $\mathrm{L}$ & -30 & 14 & 4 & 2.61 \\
\hline & Putamen & $\mathrm{L}$ & -24 & 4 & -2 & 2.61 \\
\hline & Inferior frontal gyrus, pars opercularis & $\mathrm{R}$ & 62 & 14 & 14 & 2.54 \\
\hline & Thalamus & $\mathrm{L}$ & -22 & -32 & 4 & 2.53 \\
\hline
\end{tabular}




\begin{tabular}{lllrrrr} 
& Hippocampus & L & -22 & -36 & 4 & 2.5 \\
\hline $\begin{array}{l}\text { Main effect of } \\
\text { Gender (Male }>\end{array}$ & Lingual gyrus & Medial & 4 & -78 & 2 & 2.74 \\
Female) & Occipital pole & $\mathrm{L}$ & -20 & -98 & 18 & 2.74 \\
& Precuneus & $\mathrm{R}$ & 10 & -78 & 46 & 2.7 \\
& Lateral occipital cortex & $\mathrm{L}$ & -20 & -66 & 38 & 2.69 \\
& Angular gyrus & $\mathrm{L}$ & -44 & -52 & 46 & 2.68 \\
& Anterior cingulate cortex & Medial & 2 & 10 & 42 & 2.61 \\
& Middle frontal gyrus & $\mathrm{L}$ & -40 & 38 & 32 & 2.57 \\
& Frontal pole & $\mathrm{L}$ & -48 & 42 & 14 & 2.54 \\
\hline
\end{tabular}
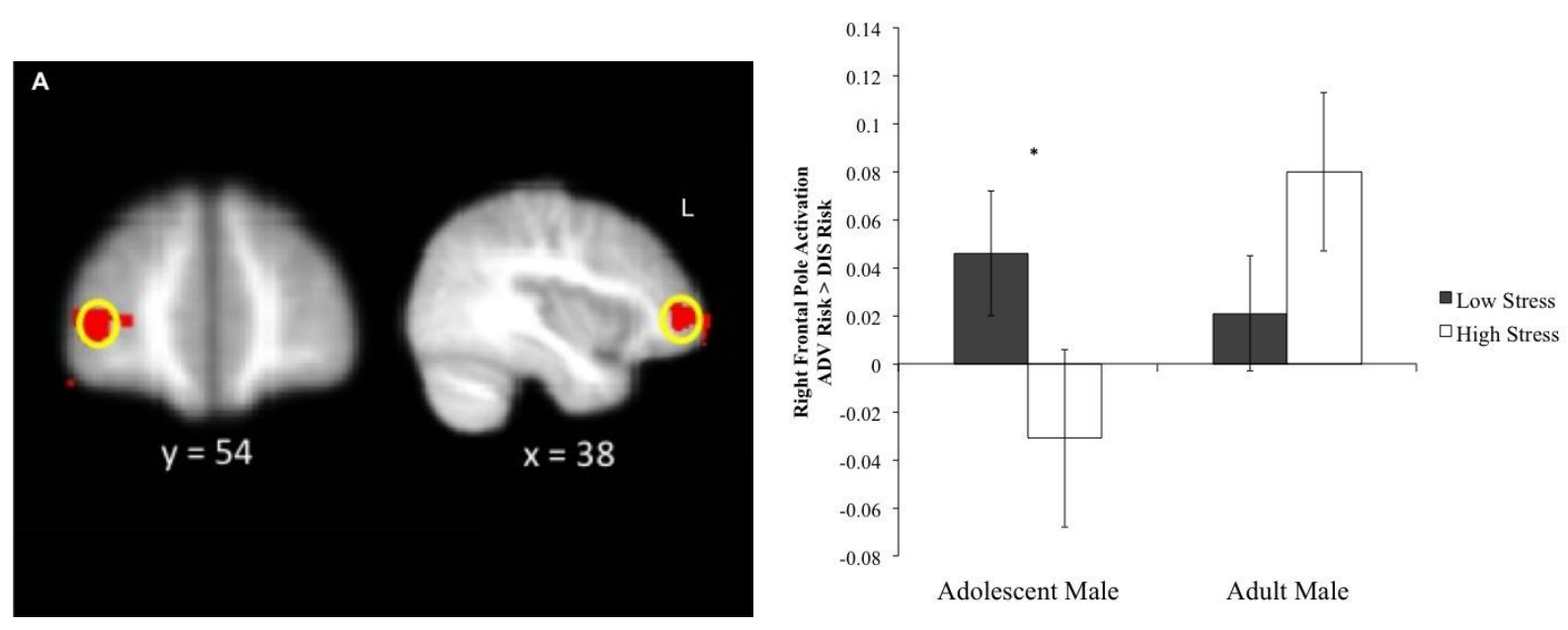

Figure 5. (A) Stress $x$ Age $x$ Gender interaction in the right frontal pole $(x=38, y=54, z=4)$ for ADV Risk > DIS Risk contrast. (B) Adolescent males showed a decrease in right frontal pole activation from low stress to high stress when taking ADV Risk > DIS Risk while adult males showed a trend towards an increase in right frontal pole activation from low stress to high stress when taking ADV Risk > DIS Risk.. 


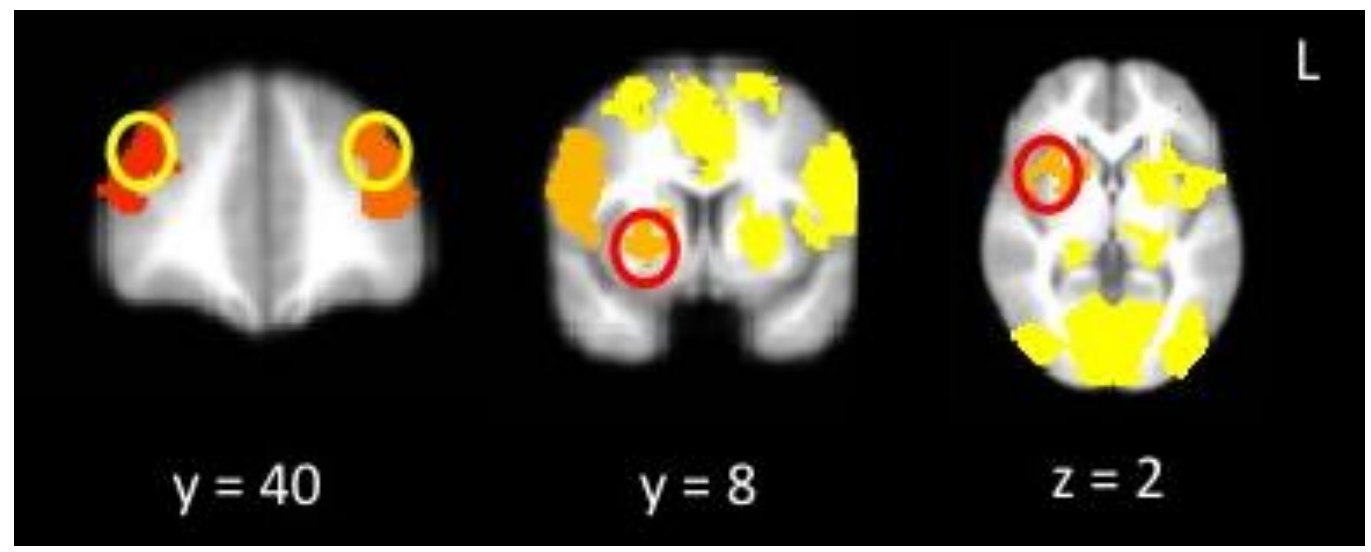

Figure 6. For ADV Risk > DIS Risk contrast, averaged across stress, age and gender, mean activation was found in bilateral frontal pole (left: $x=-40, y=40, z=30$, right: $x=50, y=42, z$ $=18)$, right putamen $(x=24, y=8, z=4)$, and right anterior insula $(x=38, y=16, z=2)$.

\subsubsection{Advantageous risks versus EQEV risks}

Within-subjects comparison of stress states revealed no significant effects. The main effects of ADV Risk > EQEV Risk contrast are listed in Table 4. No significant effects were found for the reverse contrast (EQEV Risk > ADV Risk).

\subsubsection{Disadvantageous risks versus EQEV risks}

No significant regions of activation were found for either DIS Risk > EQEV Risk or EQEV Risk > DIS Risk contrast. There were also no effects of stress or group.

\subsection{Salivary Cortisol \& fMRI Correlations}

Multiple regression analyses were conducted to determine how stress-related cortisol reactivity related to neural sensitivity to EV. Parameter estimates of regions that showed significant effects of stress (i.e., left caudate, left IFG, and right frontal pole) were regressed on cortisol difference. There were no significant associations between cortisol difference under high stress and activation in these regions.

\section{7 fMRI \& Behavior Correlations}


Multiple regression analyses were conducted to determine how neural sensitivity to EV risks was related to risky decision-making. The percentage of risks taken at each level of EV was regressed on right frontal pole activation for adult males, adolescent males, adult females, and adolescent females. Results revealed that increasing right frontal pole activation for ADV Risk > DIS Risk under high stress was associated with decreasing EQEV risks under high stress for adult males $(t(37)=-2.166, p=.037, B=-102.052)$ and adult females $(t(37)=-2.218, p=.033$, $B=-153.08$ ) (Figure 7). This association was not found for adolescent males $(p=.221)$ or adolescent females $(p=.949)$. Right frontal pole activation under low stress was not associated with EQEV risks under low stress for adult males $(p=.135)$, adult females $(p=.073)$, adolescent males $(p=.477)$, or adolescent females $(p=.682)$. Right frontal pole activation for ADV Risk > DIS Risk was not associated with ADV risks or DIS risks under high stress or low stress. Left caudate and left IFG activation for ADV Risk > DIS Risk under low or high stress were not associated with ADV risks, DIS risks, or EQEV risks taken under low or high stress, respectively. 


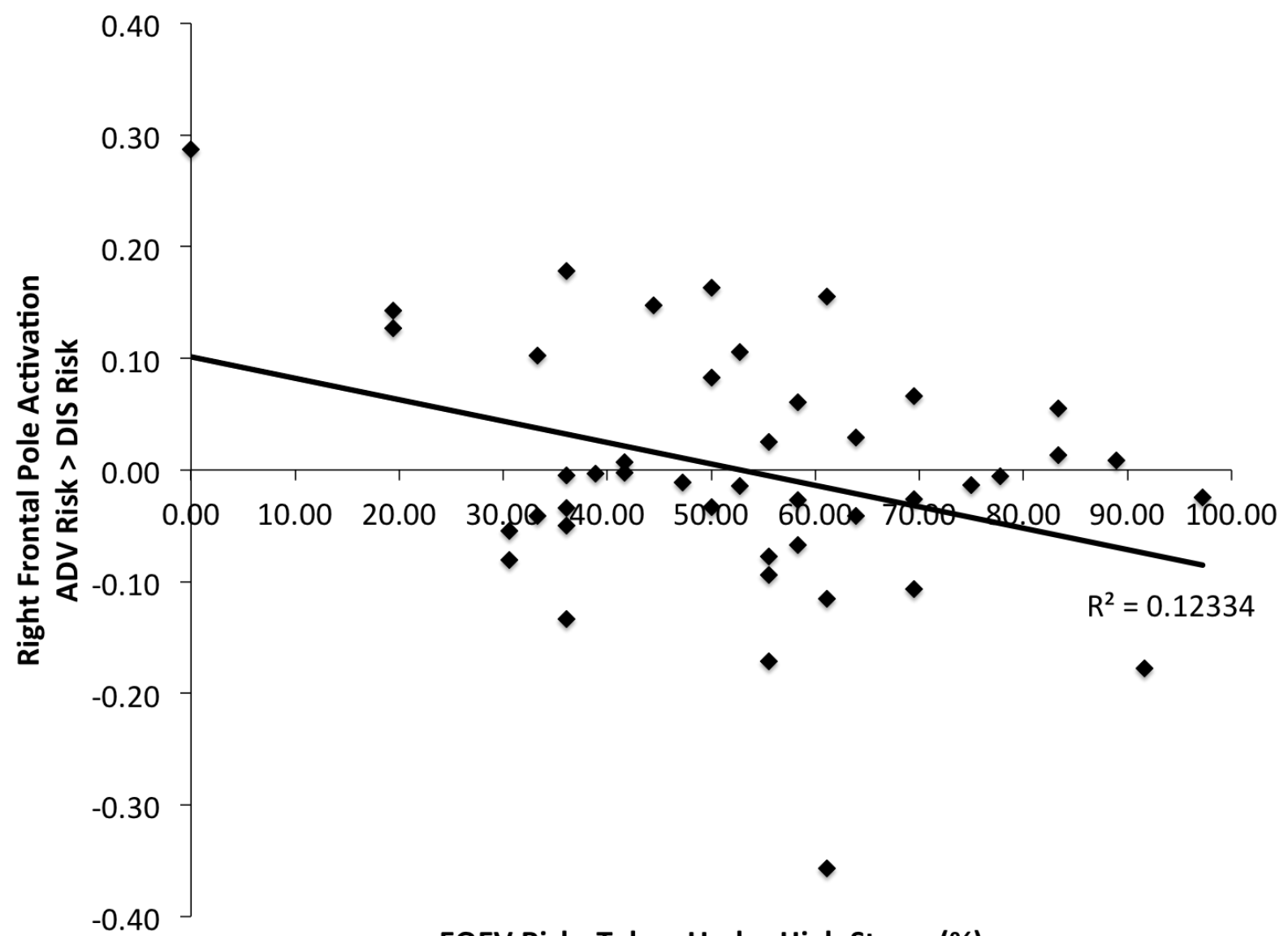

EQEV Risks Taken Under High Stress (\%)

Figure 7. Increasing right frontal pole activation for ADV Risk > DIS Risk under high stress was associated with decreasing EQEV risks under high stress.

\section{Discussion}

The goal of the present study was to examine developmental differences between adolescents and adults in the role of stress on risky decision-making and the associated neural correlates. Examining this question is important because the neurobiological targets of stress include regions implicated in risky decision-making and cognitive processing. These regions are also those that undergo significant developmental maturation during adolescence (Galván, 2013; Steinberg, 2008). 
We used a methodological approach that combined EMA methods to assess individuals' naturalistic, daily experiences of stress with neuroimaging that captured the same individuals' brain under a condition of low stress and under a condition of high stress, which allowed us to examine how stress within a person might alter behavior and neurobiology. We also used a risky decision-making task that allowed us to examine risk-taking at different levels of expected value (EV) and assess adolescents' decision-making patterns for risks that were advantageous (ADV), disadvantageous (DIS), and risks that were of equal expected value (EQEV), and how these patterns were modified by stress.

The present study showed that acute, normative self-reported stress was associated with altered patterns of risky decision-making in adolescent males and adult males while stress did not affect risky decision-making patterns in adolescent and adult females. Specifically, under low stress, adolescent males took more ADV risks than both DIS risks and EQEV risks, and more EQEV risks than DIS risks, indicating that they were sensitive to EV and modified risk-taking behavior based on these evaluations of EV. Similar patterns were also observed in adult males under low stress; however, the difference between ADV risks and DIS risks was greater in adult males than adolescent males, indicating that adult males generally showed better modification of risk-taking behavior as a function of EV. Under high stress, adolescent males took more ADV risk relative to low stress, which was more similar to adult males' behavior for ADV risks, with no stress-related changes in the proportion of DIS risks and EQEV risks taken. Moreover, under high stress, adolescent and adult males earned comparable amounts of money, which suggests an intriguing possibility: that the stress-related increase in ADV risks in adolescent males was indeed advantageous. Adult males, on the other hand, showed a decrease in EQEV risks taken under high stress relative to low stress with no stress-related changes in ADV risks and DIS 
risks, which could be due to ceiling and floor effects, respectively. Despite similar sensitivity to EV between DIS decisions and EQEV decisions (indicated by similar slopes) among adolescent males and adult males, adolescent males took more DIS risks compared to adult males under high stress. These results suggest that adolescent males may be as capable as adult males in differentiating between risks that would be advantageous and disadvantageous over accumulated trials. However, despite understanding that a risk could lead to disadvantageous outcomes or even to no differences in outcomes (i.e., EQEV trials), adolescent males continued to take risks while adult males reduced risk-taking in those contexts when stressed. Interestingly, adult males did not show an overall improvement in earnings under high stress compared to low stress conditions and even displayed a decrease in earnings (though not statistically significant), suggesting that perhaps the stress-related decrease in EQEV risks was not necessarily the more advantageous choice.

Acute stress was also associated with altered patterns of brain activation when making risky decisions between adolescent males and adult males, which predicted differences in risktaking behavior. Under low stress, adolescent males and adult males showed similar right frontal pole activation when taking ADV risks compared to DIS risks (ADV Risk > DIS Risk contrast). Under high stress, relative to low stress, adolescent males showed a significant decrease in right frontal pole activation when taking ADV risks compared to DIS risks while adult males showed no significant stress-related change in right frontal pole activation. Moreover, increasing right frontal pole activation under high stress was associated with decreasing EQEV risks under high stress in adult males. These patterns of results indicate that the adult stress response might entail maintenance of PFC function under stress to decrease risk-taking for risks that may not lead to better outcomes in the long run (i.e., EQEV risks). Adolescent males showed a decrease in PFC 
function when deciding to take risks while also showing no reduction in the amount of EQEV risk or DIS risks taken as a function of stress. This result suggests that the stress-related changes in decision-making patterns observed in adolescent males relative to their adult counterparts may have been a result of dampened engagement of frontal regions under stress, perhaps leading to greater reliance on subcortical systems when making risky decisions under stress in adolescents, as there were no significant differences in subcortical activation between adolescent and adult males.

The frontal pole is a region related to decision-making in humans and also in monkeys, but the structural and functional connectivity between the frontal pole and other regions in the decision-making circuit remain less understood. Diffusion tensor imaging studies that assessed structural connectivity have found that the frontal pole is structurally connected to the lateral PFC, the ipsilateral pallidum and putamen, and the pons (Liu et al., 2013; Moayedi, Salomons, Dunlop, Downar, \& Davis, 2014). Functional connectivity analyses suggest that this lateral frontal pole region is positively coupled with dorsolateral PFC and negatively coupled with the ACC, ventromedial PFC, the ventral striatum, and temporal cortex (Liu et al., 2013; Moayedi et al., 2014; Neubert, Mars, Sallet, \& Rushworth, 2015; Ray et al., 2015) and has been implicated in guiding attention and behavior related to goal-directed processing (Orr, Smolker, \& Banich, 2015). Yet, frontal pole lesion studies in monkeys (Boschin \& Buckley, 2015; Mansouri, Buckley, Mahboubi, \& Tanaka, 2015) and fMRI studies in humans (Boorman, Behrens, Woolrich, \& Rushworth, 2009; Daw, O’Doherty, Dayan, Dolan, \& Seymour, 2006) suggest that the frontal pole might function to redistribute cognitive resources away from the current goal to encourage exploration and evaluation of other potential goals and opportunities. In other words, when making decisions under uncertainty, the frontal pole evaluates alternative choices and 
balances the switch between dopaminergic- and striatal-related exploitation and exploration, which requires overriding exploitative tendencies. Though the complexity and design of the Cups task precludes clear categorization of exploitative and exploratory choices to confirm this hypothesis, that one needs to consider dynamic factors such as gains vs. losses, expected value of the choices, and the degree of reward processing from prior experiences need to be taken into account when making decisions throughout the task suggests that adaptive decision-making requires considerable context-dependent evaluation and switching between making safe and risky choices that might depend on the frontal pole, a region that shows protracted development through adolescence.

These behavioral and neural findings are consistent with the hypothesis that stress-related cortisol increases in dopamine release on an already saturated adolescent dopaminergic system could create an influx of dopamine in response to risk and reward. Excess binding of D1 receptors by dopamine in the PFC could lead to suppression of PFC function, further subverting maturing regulatory functions and leading to greater reward-driven risky behaviors in adolescents compared to adults. Indeed, in adolescent rats, administration of D1 agonists increased preference for cocaine-associated environments while D1 antagonists blocked this effect (Brenhouse et al., 2008). Animal studies also showed that adolescent male rats exhibited prolonged release of glucocorticoids in response to acute stress relative to adult males as a result of an immature negative feedback system (McCormick \& Mathews, 2007; Romeo et al., 2014; Vázquez \& Akil, 1993), which could prolong and amplify the neural effects of stress in adolescent males. In the current study, we found that cortisol reactivity to stress was associated with increased EQEV risks under high stress. However, we did not find that cortisol was related to neural activation in regions that showed an effect of stress, which could be due to the 
imprecision of our cortisol sampling both within and between participants as well as differences in recovery that we were not able to measure. This precluded us from empirically testing neural activation as a mediator between cortisol and risk-taking.

We also found that stress amplified gender differences in risky decision-making and that the effects were stronger in adults than in adolescents. Namely, we found that acute stress altered risky decision-making behavior in males, but there were no effects of stress on risky decisionmaking in females. These findings are consistent with previous reports that stress led to divergent patterns of risky decision-making in males and females (Lighthall et al., 2012, 2009). However, while previous studies found that stressed women took less risks (Lighthall et al., 2009) and were slower to make decisions (Lighthall et al., 2012) than women in the control group and men under stress, our study did not yield any within-subjects effects of stress for females. These disparate findings could be due to differences in the decision-making task used between the studies, as the BART and Cups task may assess different risky decision-making processes. Specifically, the Cups task provides explicit information regarding the probability of receiving a reward and the value of the reward, allowing participants to calculate EV and objectively decide which risks are advantageous to take and which are not. Knowing that it is more advantageous to take a risk and then doing so while refraining from taking disadvantageous risks shows rational decision-making capabilities. On the other hand, the BART task, without access to explicit information about probability and outcome, may perhaps reflect participants' sensation seeking and risk propensity rather than rational decision-making capacity. There were also differences in how stress was induced in participants such that Lighthall et al. (2012) used a cold pressor stress task to induce stress while the current study utilized participants' self-reported ratings of daily stress in a within-subjects design. While our findings contribute to knowledge 
about gender-stress interactions in risky decision-making, further research is needed to better understand the mechanisms that underlie these interactions between stress and gender on risktaking and decision-making.

Although stress might affect decision-making under uncertainty differently in men and women, research suggests that these gender differences in stress effects might be domainspecific to risk-taking and do not generalize to other cognitive domains such as learning (Lighthall, Gorlick, Schoeke, Frank, \& Mather, 2013; Petzold, Plessow, Goschke, \& Kirschbaum, 2010) and memory (Buchanan \& Lovallo, 2001). For instance, on a probabilistic reinforcement-learning task where participants were asked to learn probabilistic associations between visual cues and different types of feedback through trial and error, individuals who were stressed showed better learning from positive feedback and worse learning from negative feedback (Lighthall et al., 2013; Petzold et al., 2010), demonstrating that stress enhanced learning about positive outcomes while diminishing learning about negative outcomes. Moreover, the effects of stress on reinforcement learning were similar for both males and females, suggesting that gender differences in stress effects might be domain-specific to risky decision-making.

The current study demonstrates the feasibility of combining daily diary approaches with fMRI to determine the effects of stress on adolescent cognition. The stress manipulation in this study is a significant and novel contribution. This approach allowed us to reduce participant variability in reactions to laboratory-based stressors and examine the effects of real-life stress on decision-making. However, there are a few limitations to note. First, the non-experimental nature of our stress induction limits our ability to precisely characterize the role of cortisol in stressrelated differences in neural response. Nonetheless, our results suggest that our stress 
manipulation is robust and captures stress-related differences in risky decision-making behavior and its neural correlates. Second, there may be differences in cerebral blood flow (CBF) in response to stress both within-individuals across the two stress scans and between adolescents and adults that are unaccounted for in our study (Buxton, 2010).

Conclusion

The current study demonstrated that naturalistic daily stress alters risky decision-making among adolescent and adult males while stress did not affect risky decision-making in adolescent and adult females. In adult males, acute stress led to decreased risk-taking for risks that were not advantageous while risk-taking for advantageous risks remained high. In contrast, acute stress led to greater risk-taking for risks that were advantageous in adolescent males, which was more similar to adult males' behavior, while there were no changes in behavior for risks that were not advantageous, which remained higher than that observed in adult males. These developmental differences in stress-related behavior appear to be supported by developmentally unique differences in PFC function in response to stress such that adolescent males showed decreased PFC function under stress while adult males maintained PFC function when making risky decisions under stress, which resulted in decreased risk-taking for risks that were not advantageous. 


\section{References}

Abercrombie, E. (1989). Differential effect of stress on in vivo dopamine release in striatum, nucleus accumbens, and medial frontal cortex. Journal of Neurochemistry, 52(5), 16551658.

Almeida, D. M., McGonagle, K., \& King, H. (2009). Assessing daily stress processes in social surveys by combining stressor exposure and salivary cortisol. Biodemography and Social Biology, 55(2), 219-237. http://doi.org/10.1080/19485560903382338

Andersen, S. L., Thompson, A. P., Krenzel, E., \& Teicher, M. H. (2002). Pubertal changes in gonadal hormones do not underlie adolescent dopamine receptor overproduction. Psychoneuroendocrinology, 27(6), 683-691. http://doi.org/10.1016/S0306-4530(01)000695

Armbruster, D., Mueller, A., Strobel, A., Lesch, K.-P., Brocke, B., \& Kirschbaum, C. (2012). Children under stress - COMT genotype and stressful life events predict cortisol increase in an acute social stress paradigm. The International Journal of Neuropsychopharmacology, 15(09), 1229-1239. http://doi.org/10.1017/S1461145711001763

Arnsten, A. F., \& Goldman-Rakic, P. S. (1998). Noise stress impairs prefrontal cortical cognitive function in monkeys: evidence for a hyperdopaminergic mechanism. Archives of General Psychiatry, 55(4), 362-368. http://doi.org/10.1001/archpsyc.55.4.362

Bolger, N., Davis, A., \& Rafaeli, E. (2003). Diary methods: Capturing life as it is lived. Annual Review of Psychology, 54(1), 579-616. http://doi.org/10.1146/annurev.psych.54.101601.145030

Boorman, E. D., Behrens, T. E. J., Woolrich, M. W., \& Rushworth, M. F. S. (2009). How Green Is the Grass on the Other Side? Frontopolar Cortex and the Evidence in Favor of Alternative 
Courses of Action. Neuron, 62(5), 733-743. http://doi.org/10.1016/j.neuron.2009.05.014

Boschin, E. A., \& Buckley, M. J. (2015). Differential contributions of dorsolateral and frontopolar cortices to working memory processes in the primate. Frontiers in Systems Neuroscience, 9(October), Article 144. http://doi.org/10.3389/fnsys.2015.00144

Braams, B. R., van Duijvenvoorde, A. C. K., Peper, J. S., \& Crone, E. A. (2015). Longitudinal Changes in Adolescent Risk-Taking: A Comprehensive Study of Neural Responses to Rewards, Pubertal Development, and Risk-Taking Behavior. Journal of Neuroscience, 35(18), 7226-7238. http://doi.org/10.1523/JNEUROSCI.4764-14.2015

Brenhouse, H. C., Sonntag, K. C., \& Andersen, S. L. (2008). Transient D1 dopamine receptor expression on prefrontal cortex projection neurons: Relationship to enhanced motivational salience of drug cues in adolescence. Journal of Neuroscience, 28(10), 2375-2382. http://doi.org/10.1523/JNEUROSCI.5064-07.2008

Buchanan, T. W., \& Lovallo, W. R. (2001). Enhanced memory for emotional material following stress-level cortisol treatment in humans. Psychoneuroendocrinology, 26(3), 307-317. http://doi.org/10.1016/S0306-4530(00)00058-5

Burnett, S., Bault, N., Coricelli, G., \& Blakemore, S.-J. (2010). Adolescents' heightened riskseeking in a probabilistic gambling task. Cognitive Development, 25(2), 183-196. http://doi.org/10.1016/j.cogdev.2009.11.003

Buxton, R. B. (2010). Interpreting oxygenation-based neuroimaging signals: the importance and the challenge of understanding brain oxygen metabolism. Frontiers in Neuroenergetics, 2(June), 1-16. http://doi.org/10.3389/fnene.2010.00008

Cauffman, E., Shulman, E. P., Steinberg, L., Claus, E., Banich, M. T., Graham, S., \& Woolard, J. (2010). Age differences in affective decision making as indexed by performance on the 
Iowa Gambling Task. Developmental Psychology, 46(1), 193-207. http://doi.org/10.1037/a0016128

Chen, E., Cohen, S., \& Miller, G. E. (2010). How low socioeconomic status affects 2-year hormonal trajectories in children. Psychological Science, 21(1), 31-37. http://doi.org/10.1177/0956797609355566

Clements, A. D. (2012). Salivary cortisol measurement in developmental research: Where do we go from here? Developmental Psychobiology, 55(3), 205-220. http://doi.org/10.1002/dev.21025

Cohen, S., Schwartz, J. E., Epel, E., Kirschbaum, C., Sidney, S., \& Seeman, T. (2006). Socioeconomic status, race, and diurnal cortisol decline in the Coronary Artery Risk Development in Young Adults (CARDIA) Study. Psychosomatic Medicine, 68(1), 41-50. http://doi.org/10.1097/01.psy.0000195967.51768.ea

Dahl, R. E. (2004). Adolescent brain development: A period of vulnerabilities and opportunities - Keynote Address. Annals of the New York Academy of Sciences, 1021, 1-22. http://doi.org/10.1196/annals.1308.001

Dahl, R. E., \& Gunnar, M. R. (2009). Heightened stress responsiveness and emotional reactivity during pubertal maturation: implications for psychopathology. Development and Psychopathology, 21(1), 1-6. http://doi.org/10.1017/S0954579409000017

Daw, N. D., O’Doherty, J. P., Dayan, P., Dolan, R. J., \& Seymour, B. (2006). Cortical substrates for exploratory decisions in humans. Nature, 441(7095), 876-9. http://doi.org/10.1038/nature04766

Desantis, A. S., Kuzawa, C. W., \& Adam, E. K. (2015). Developmental origins of flatter cortisol rhythms: socioeconomic status and adult cortisol activity. American Journal of Human 
Biology, 27(4), 458-467. http://doi.org/10.1002/ajhb.22668

Galván, A. (2013). The teenage brain: Sensitivity to rewards. Current Directions in Psychological Science, 22(2), 88-93. http://doi.org/10.1177/0963721413480859

Galván, A., Hare, T. A., Parra, C. E., Penn, J., Voss, H., Glover, G., \& Casey, B. J. (2006). Earlier development of the accumbens relative to orbitofrontal cortex might underlie risktaking behavior in adolescents. Journal of Neuroscience, 26(25), 6885-6892. http://doi.org/10.1523/JNEUROSCI.1062-06.2006

Galván, A., \& McGlennen, K. M. (2012). Daily stress increases risky decision-making in adolescents: a preliminary study. Developmental Psychobiology, 54(4), 433-40. http://doi.org/10.1002/dev.20602

Gibbs, S. E. B., \& D’Esposito, M. (2005). A functional MRI study of the effects of bromocriptine, a dopamine receptor agonist, on component processes of working memory. Psychopharmacology, 180(4), 644-53. http://doi.org/10.1007/s00213-005-0077-5

Hajnal, A., Smith, G. P., \& Norgren, R. (2004). Oral sucrose stimulation increases accumbens dopamine in the rat. American Journal of Physiology. Regulatory, Integrative and Comparative Physiology, 286(1), R31-R37. http://doi.org/10.1152/ajpregu.00282.2003

Hellhammer, D. H., Wüst, S., \& Kudielka, B. M. (2009). Salivary cortisol as a biomarker in stress research. Psychoneuroendocrinology, 34(2), 163-171. http://doi.org/10.1016/j.psyneuen.2008.10.026

Kogler, L., Mueller, V. I., Chang, A., Eickhoff, S. B., Gur, R. C., \& Derntl, B. (2013). Psychosocial vs . physiological - an ALE meta-analysis on the neural correlates of stress reactions. NeuroImage, 119, 1328. http://doi.org/10.1016/j.neuroimage.2015.06.059

Lamm, C., Benson, B. E., Guyer, A. E., Perez-Edgar, K., Fox, N. A., Pine, D. S., \& Ernst, M. 
(2014). Longitudinal study of striatal activation to reward and loss anticipation from midadolescence into late adolescence/early adulthood. Brain and Cognition, 89, 51-60. http://doi.org/10.1016/j.bandc.2013.12.003

Levin, I. P., \& Hart, S. S. (2003). Risk preferences in young children: Early evidence of individual differences in reaction to potential gains and losses. Journal of Behavioral Decision Making, 16(October), 397-413. http://doi.org/10.1002/bdm.453

Levin, I. P., Weller, J. A., Pederson, A. A., \& Harshman, L. A. (2007). Age-related differences in adaptive decision making: Sensitivity to expected value in risky choice. Judgment and Decision Making, 2(4), 225-233. Retrieved from http://journal.sjdm.org/7404/jdm7404.htm

Lidow, M. S., \& Rakic, P. (1992). Scheduling of monoaminergic neurotransmitter receptor expression in the primate neocortex during postnatal development. Cerebral Cortex (New York, N.Y. : 1991), 2(5), 401-16. http://doi.org/10.1093/cercor/2.5.401

Lighthall, N. R., Gorlick, M. A., Schoeke, A., Frank, M. J., \& Mather, M. (2013). Stress modulates reinforcement learning in younger and older adults. Psychol. Aging, 28(1), 3546. http://doi.org/10.1037/a0029823.Stress

Lighthall, N. R., Mather, M., \& Gorlick, M. a. (2009). Acute stress increases sex differences in risk seeking in the balloon analogue risk task. PloS One, 4(7), e6002. http://doi.org/10.1371/journal.pone.0006002

Lighthall, N. R., Sakaki, M., Vasunilashorn, S., Nga, L., Somayajula, S., Chen, E. Y., ... Mather, M. (2012). Gender differences in reward-related decision processing under stress. Social Cognitive and Affective Neuroscience, 7(4), 476-84. http://doi.org/10.1093/scan/nsr026

Liu, H., Qin, W., Li, W., Fan, L., Wang, J., Jiang, T., \& Yu, C. (2013). Connectivity-based parcellation of the human frontal pole with diffusion tensor imaging. The Journal of 
Neuroscience: The Official Journal of the Society for Neuroscience, 33(16), 6782-90. http://doi.org/10.1523/JNEUROSCI.4882-12.2013

Mansouri, F. A., Buckley, M. J., Mahboubi, M., \& Tanaka, K. (2015). Behavioral consequences of selective damage to frontal pole and posterior cingulate cortices. Proceedings of the National Academy of Sciences of the United States of America, 112(29), E3940-3949. http://doi.org/10.1073/pnas.1422629112

McCormick, C. M., \& Mathews, I. Z. (2007). HPA function in adolescence: role of sex hormones in its regulation and the enduring consequences of exposure to stressors. Pharmacology, Biochemistry, and Behavior, 86(2), 220-33. http://doi.org/10.1016/j.pbb.2006.07.012

Moayedi, M., Salomons, T. V., Dunlop, K. A. M., Downar, J., \& Davis, K. D. (2014). Connectivity-based parcellation of the human frontal polar cortex. Brain Structure and Function, 220(5), 2603-2616. http://doi.org/10.1007/s00429-014-0809-6

Neubert, F.-X., Mars, R. B., Sallet, J., \& Rushworth, M. F. S. (2015). Connectivity reveals relationship of brain areas for reward-guided learning and decision making in human and monkey frontal cortex. Proceedings of the National Academy of Sciences of the United States of America, 1-10. http://doi.org/10.1073/pnas.1410767112

Orr, J. M., Smolker, H. R., \& Banich, M. T. (2015). Organization of the human frontal pole revealed by large-scale DTI-based connectivity: Implications for control of behavior. PLoS ONE, 10(5), 1-23. http://doi.org/10.1371/journal.pone.0124797

Pabst, S., Brand, M., \& Wolf, O. T. (2013). Stress effects on framed decisions: there are differences for gains and losses. Frontiers in Behavioral Neuroscience, 7(October), 142. http://doi.org/10.3389/fnbeh.2013.00142 
Petzold, A., Plessow, F., Goschke, T., \& Kirschbaum, C. (2010). Stress reduces use of negative feedback in a feedback-based learning task. Behavioral Neuroscience, 124(2), 248-255. http://doi.org/10.1037/a0018930

Porcelli, A. J., \& Delgado, M. R. (2009). Acute stress modulates risk taking in financial decision making. Psychological Science, 20(3), 278-283. http://doi.org/10.1111/j.14679280.2009.02288.x

Pruessner, J. C., Champagne, F., Meaney, M. J., \& Dagher, A. (2004). Dopamine release in response to a psychological stress in humans and its relationship to early life maternal care: a positron emission tomography study using [11C]raclopride. The Journal of Neuroscience : The Official Journal of the Society for Neuroscience, 24(11), 2825-2831. http://doi.org/10.1523/JNEUROSCI.3422-03.2004

Pruessner, J. C., Dedovic, K., Khalili-Mahani, N., Engert, V., Pruessner, M., Buss, C., ... Lupien, S. (2008). Deactivation of the limbic system during acute psychosocial stress: Evidence from positron emission tomography and functional magnetic resonance imaging studies. Biological Psychiatry, 63(2), 234-240. http://doi.org/10.1016/j.biopsych.2007.04.041

Putman, P., Antypa, N., Crysovergi, P., \& van der Does, W. A. J. (2010). Exogenous cortisol acutely influences motivated decision making in healthy young men. Psychopharmacology, 208(2), 257-63. http://doi.org/10.1007/s00213-009-1725-y

Rahdar, A., \& Galván, A. (2014). The cognitive and neurobiological effects of daily stress in adolescents. NeuroImage, 92, 267-73. http://doi.org/10.1016/j.neuroimage.2014.02.007

Ray, K. L., Zald, D. H., Bludau, S., Riedel, M. C., Bzdok, D., Yanes, J., ... Laird, A. R. (2015). Co-activation based parcellation of the human frontal pole. NeuroImage, 123, 200-211. 
http://doi.org/10.1016/j.neuroimage.2015.07.072

Romeo, R. D., Minhas, S., Svirsky, S. E., Hall, B. S., Savenkova, M., \& Karatsoreos, I. N.

(2014). Pubertal shifts in adrenal responsiveness to stress and adrenocorticotropic hormone in male rats. Psychoneuroendocrinology, 42, 146-152.

http://doi.org/10.1016/j.psyneuen.2014.01.016

Rougé-Pont, F., Deroche, V., Le Moal, M., \& Piazza, P. V. (1998). Individual differences in stress-induced dopamine release in the nucleus accumbens are influenced by corticosterone. European Journal of Neuroscience, 10(12), 3903-3907. http://doi.org/10.1046/j.14609568.1998.00438.x

Seeman, P., Bzowej, N. H., Guan, H. C., Bergeron, C., Becker, L. E., Reynolds, G. P., ... Watanabe, S. (1987). Human brain dopamine receptors in children and aging adults. Synapse (New York, N.Y.), 1(5), 399-404. http://doi.org/10.1002/syn.890010503

Starcke, K., Wolf, O. T., Markowitsch, H. J., \& Brand, M. (2008). Anticipatory stress influences decision making under explicit risk conditions. Behavioral Neuroscience, 122(6), 13521360. http://doi.org/10.1037/a0013281

Steinberg, L. (2008). A Social Neuroscience Perspective on Adolescent Risk-Taking. Developmental Review, 28(1), 78-106. http://doi.org/10.1016/j.dr.2007.08.002

Strahler, J., Mueller, A., Rosenloecher, F., Kirschbaum, C., \& Rohleder, N. (2010). Salivary $\alpha-$ amylase stress reactivity across different age groups. Psychophysiology, 47(3), 587-595. http://doi.org/10.1111/j.1469-8986.2009.00957.x

Stroud, L. R., Foster, E., Papandonatos, G. D., Handwerger, K., Granger, D. a, Kivlighan, K. T., \& Niaura, R. (2009). Stress response and the adolescent transition: Performance versus peer rejection stressors. Development and Psychopathology, 21(1), 47-68. 
http://doi.org/10.1017/S0954579409000042.Stress

van Leijenhorst, L., Zanolie, K., van Meel, C. S., Westenberg, P. M., Rombouts, S. a R. B., \& Crone, E. a. (2010). What motivates the adolescent? Brain regions mediating reward sensitivity across adolescence. Cerebral Cortex (New York, N.Y. : 1991), 20(1), 61-9. http://doi.org/10.1093/cercor/bhp078

Vázquez, D. M., \& Akil, H. (1993). Pituitary-adrenal response to ether vapor in the weanling animal: characterization of the inhibitory effect of glucocorticoids on adrenocorticotropin secretion. Pediatric Research, 34(5), 646-653. http://doi.org/10.1203/00006450199311000-00017

Vijayraghavan, S., Wang, M., Birnbaum, S. G., Williams, G. V, \& Arnsten, A. F. T. (2007). Inverted-U dopamine D1 receptor actions on prefrontal neurons engaged in working memory. Nature Neuroscience, 10(3), 376-384. http://doi.org/10.1038/nn1846

Xue, G., Lu, Z., Levin, I. P., \& Bechara, A. (2013). An fMRI study of risk-taking following wins and losses: Implications for the gambler's fallacy. Human Brain Mapping, 18(9), 11991216. http://doi.org/10.1002/hbm.21015

Xue, G., Lu, Z., Levin, I. P., Weller, J. A., Li, X., \& Bechara, A. (2009). Functional dissociations of risk and reward processing in the medial prefrontal cortex. Cerebral Cortex (New York, N.Y. : 1991), 19(5), 1019-27. http://doi.org/10.1093/cercor/bhn147

Zahrt, J., Taylor, J. R., Mathew, R. G., \& Arnsten, A. F. T. (1997). Supranormal Stimulation of D 1 Dopamine Receptors in the Rodent Prefrontal Cortex Impairs Spatial Working Memory Performance, $17(21), 8528-8535$. 